\title{
NADM Conceptual Model 1.0- A Conceptual Model for Geologic Map Information
}

By North American Geologic Map Data Model (NADM) Steering Committee Data Model Design Team

Any use of trade, firm, or product names is for descriptive purposes only and does not imply endorsement by the U.S. Government

\section{Open-File Report 2004-1334}

This document should be cited as:

North American Geologic Map Data Model Steering Committee, 2004, NADM Conceptual Model 1.0-A conceptual model for geologic map information: U.S. Geological Survey Open-File Report 2004-1334, 58 p., accessed online at URL http://pubs.usgs.gov/of/2004/1334. Also published as Geological Survey of Canada Open File 4737, 1 CD-ROM.

\section{U.S. Department of the Interior U.S. Geological Survey}




\section{U.S. Department of the Interior \\ Gale A. Norton, Secretary}

\section{U.S. Geological Survey \\ Charles G. Groat, Director}

U.S. Geological Survey, Reston, Virginia 2004

For product and ordering information:

World Wide Web: http://www.usgs.gov/pubprod

Telephone: 1-888-ASK-USGS

For more information on the USGS - the Federal source for science about the Earth, its natural and living resources, natural hazards, and the environment:

World Wide Web: http://www.usgs.gov

Telephone: 1-888-ASK-USGS

Although this report is in the public domain, permission must be secured from the individual copyright owners to reproduce any copyrighted material contained within this report.

Cover Illustration from: Morton, D.M., Miller, F.K., Cossette, P.M., and Bovard, K.R. (2003). Preliminary geologic map of the San Bernardino 30' X 60' quadrangle, California. USGS Open File Report OF-03-293. 


\section{TABLE OF CONTENTS}

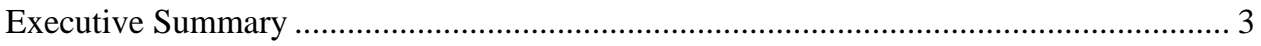

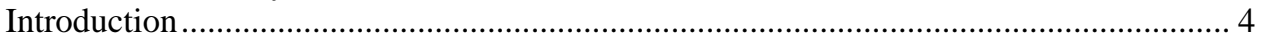

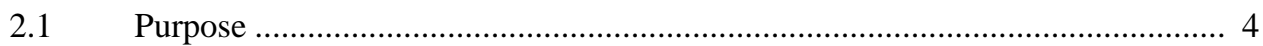

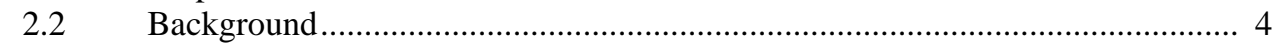

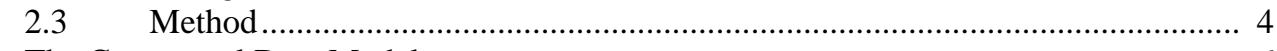

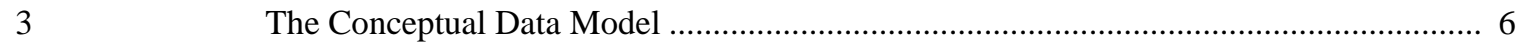

Role of geologic vocabularies

Discussion of Model elements (Plates 1-13) ................................................................ 10

$4.1 \quad$ Top-Level Geologic Objects (Plate 1) ..................................................... 10

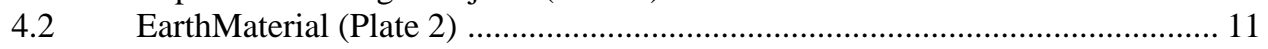

G.3 GeologicUnit (Plate 3) ........................................................................... 12

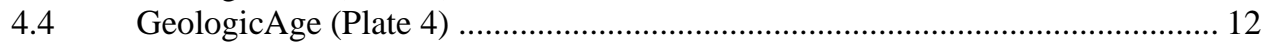

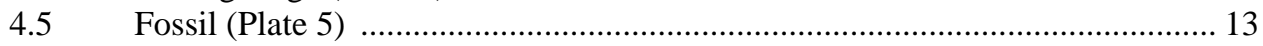

4.6 GeologicStructure (Plate 6) ....................................................................... 13

4.7 Geologic Process and Genesis (Plate 7) ........................................................ 14

G.8 GeologicRelation (Plate 8) ........................................................................... 14

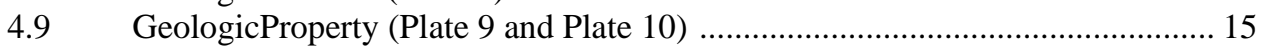

4.10 GeologicVocabulary (Plate 11)................................................................ 15

4.11 GeologicPortrayal (Plate 12) and GeologicPortrayal Component (Plate 13)... 15

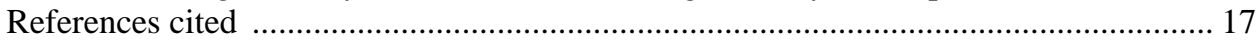

Appendix 1. Scope Notes for classes in UML model ................................................ 19

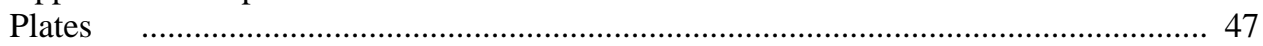

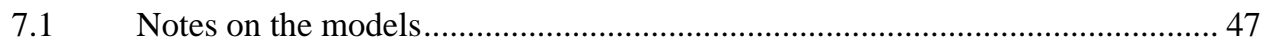




\section{EXECUTIVE SUMMARY}

The NADM Data Model Design Team was established in 1999 by the North American Geologic Map Data Model Steering Committee (NADMSC) with the purpose of drafting a geologic map data model for consideration as a standard for developing interoperable geologic map-centered databases by state, provincial, and federal geological surveys. The model is designed to be a technology-neutral conceptual model that can form the basis for a webbased interchange format using evolving information technology (e.g., XML, RDF, OWL), and guide implementation of geoscience databases in a common conceptual framework. The intended purpose is to allow geologic information sharing between geologic map data providers and users, independent of local information system implementation.

The model emphasizes geoscience concepts and relationships related to information presented on geologic maps. Design has been guided by an informal requirements analysis, documentation of existing databases, technology developments, and other standardization efforts in the geoscience and computer-science communities.

A key aspect of the model is the notion that representation of the conceptual framework (ontology) that underlies geologic map data must be part of the model, because this framework changes with time and understanding, and varies between information providers. The top level of the model distinguishes geologic concepts, geologic representation concepts, and metadata. The geologic representation part of the model provides a framework for representing the ontology that underlies geologic map data through a controlled vocabulary, and for establishing the relationships between this vocabulary and a geologic map visualization or portrayal. Top-level geologic classes in the model are Earth material (substance), geologic unit (parts of the Earth), geologic age, geologic structure, fossil, geologic process, geologic relation, and geologic event. 


\section{INTRODUCTION}

\subsection{Purpose}

The purpose of this document (hereafter referred to as North American Geologic Map Data Model--Conceptual Model 1.0, or NADM-C1) is to present a data-model standard for the description, classification, and interpretation of geologic features in digital geologic map databases.

\subsection{Background}

NADM-C1 is part of a larger effort to develop standardized methodologies for the storage, manipulation, analysis, management, and distribution of digital geologic map information. This continent-wide effort began in 1999 under the auspices of a North American Geologic Map Data Model Steering Committee (NADMSC) an oversight group composed of representatives from Canadian and American geoscience agencies. The datamodel effort has several components:

1. A conceptual model for storing digital data, and for manipulating these data in a relational and (or) object-oriented database environment (the subject of this report);

2. Standardized science language that allows geologic materials and geologic structures to be described, classified, and interpreted;

3. Software tools for entering data into the model at the front end (data-producer) and for extracting the data at the back end (data-user);

4. Methodologies and techniques for exchanging data sets having different structures and formats.

NADM-C1 builds on an earlier prototype data-model standard now referred to as North American Geologic Map Data Model (NADM) version 4.3 [Johnson and others, 1999]. NADM 4.3 was developed in response to a cooperative agreement between the Association of American State Geologists (AASG) and the U.S. Geological Survey (USGS) in 1996 at the AASG annual meeting. Release of NADM 4.3 was followed by a period of experimental implementation by various groups developing geologic map databases. The initial intent was that each group would implement the logical model presented by Johnson and others [1999]. Each individual implementation evolved to varying degrees from the NADM 4.3 model, and it soon became apparent that another effort was necessary to generalize from the existing implementations to a model that could serve as a standard. NADM-C1 represents this conceptual standard.

\subsection{Method}

The NADM Data Model Design Team (DMDT, Table 1) was established in 1999 by the North American Geologic Map Data Model Steering Committee (NADMSC) with the purpose of drafting a geologic map data model for consideration as a standard.

As a first step, DMDT asked the various groups implementing databases related to or derived from NADM 4.3 (referred to as "variants") to document their implementation, comparing and contrasting them with the NADM 4.3 model. To date, documentation for four variants has been posted on the DMDT web site. These variants are CORDLink v5.2 (Geological Survey of Canada, Pacific; Brodaric and others, 1999), Idaho Geological Survey (IGS; Stanford and others, 1999; Stanford, 2002), Arizona Geological Survey (AZGS; Richard and Orr, 2001), and NGMDB/Kentucky (U.S. Geological Survey National Geologic Map Database project and Kentucky

\footnotetext{
${ }^{1}$ Visit http://nadm-geo.org/ for information about the North American Data Model Steering Committee, its mandate, and its constituency.
} 
Geological Survey; Soller and others, 2002). This documentation has served as a resource for development of the conceptual model presented here.

As a second step, the DMDT developed a requirements analysis by analyzing several hundred queries that could be posed to a geologic map information database. The Science Language Technical Team (SLTT) of the NADMSC collected the queries in a survey of State and Federal geological survey geologists. DMDT analyzed these queries to determine the kinds of things that need to be represented in a geologic map data model. Results of this effort are summarized in DMDT [2002]. The full list of queries and analysis documents are available at http://nadm-geo.org/.

A DMDT working group consisting of Boyan Brodaric, Bruce Johnson, Stephen Richard, Peter Schweitzer, and Gerald Weisenfluh developed a draft conceptual model through a series of five working group meetings. The group developed a scheme of top-level geologic classes to model, and sequentially developed subtype hierarchies for each class and schema for the properties of the classes and relationships between them. The draft model was reviewed and revised by the entire DMDT, and submitted to the NADMSC for review and approval. The formal NADM-C1 document will be released simultaneously in early 2004 as USGS and GSC open-file reports.

Table 1. NADM Data Model Design Team membership

\begin{tabular}{|c|c|}
\hline \multicolumn{2}{|c|}{$\begin{array}{c}\text { Committee Members } \\
\text { Data Model Design Technical Team of the North American Geologic } \\
\text { Map Data Model Steering Committee }\end{array}$} \\
\hline Eric Boisvert & Geological Survey of Canada \\
\hline $\begin{array}{c}\text { Boyan Brodaric } \\
\text { (committee chair) }\end{array}$ & Geological Survey of Canada \\
\hline Jordan Hastings & University of California, Santa Barbara \\
\hline Bruce R. Johnson & U.S. Geological Survey \\
\hline James McDonald & Ohio Geological Survey \\
\hline Stephen M. Richard & Arizona Geological Survey \\
\hline Peter N. Schweitzer & U.S. Geological Survey \\
\hline Ronald R. Wahl & U.S. Geological Survey \\
\hline Gerald A Weisenfluh & Kentucky Geological Survey \\
\hline
\end{tabular}




\section{THE CONCEPTUAL DATA MODEL}

NADM-C1 is designed to be a technology-neutral conceptual model that can form the basis for a web-based interchange format using evolving information technology (e.g., XML). The intended purpose is to allow the sharing of geologic information independent of logical and physical implementations. It is a model of geoscience concepts and the relationships between them with special emphasis on concepts related to information presented on geologic maps [Richard, 1999 and submitted; Brodaric and Gahegan, 2000; Brodaric and Hastings, 2002; Soller and others, 2002]. The NADM-C1 is based on NADM 4.3, the DMDT requirements analysis, variant documentation, and other standardization efforts, technology developments, and data-sharing initiatives in the geoscience and computer-science communities. These include Environmental Systems Research Institute (ESRI) geodatabase data model development, Exploration and Mining Markup Language (XMML), Geoinformatics Initiative, Open GIS Consortium, International Standards Organization Geographic information/Geomatics (TC 211), and others.

This model has been referred to informally as NORTON during its development phase. The name derives from NORTh American geologic map ONtology. With release of version one, this model will henceforth be referred to as NADM-C1. Plates 1-13 are schematic diagrams of the model using UML notation. In the following discussion, reference to specific model elements is indicated by use of Courier font. When the discussion pertains to the geoscience aspects of the concepts, normal text is used.

The top level of the model is 'Universe', representing all concepts (Plate 1). Three subclasses of Universe are represented: GeologicConcept, Metadata, and GeologicRepresentation. GeologicConcept includes concepts specific to the domain of geoscience knowledge (Table 2). The term 'Concept' as used here represents the notion of any mental phenomena that human beings use in their internal representation of the world. Webster's dictionary [1996] uses the terms 'idea' and 'object of thought' to convey the meaning of 'concept.' GeologicRepresentation includes concepts related to the representation of geoscience knowledge, particularly through the medium of a geologic map. Table 3 summarizes the major concepts included as GeologicRepresentations. Metadata includes concepts related to documentation of specific items of data in a database (i.e. instances of classes in the model). This high-level part of the model and its relationship to other ontologies, both top level (e.g. OpenCyc, Lenat [1995]; SUMO, Pease and Niles [2002]; DOLCE, Gangemi and others [2002]; Sowa [2000]), or domain-level (e.g. ontologies for spatial or temporal concepts) is still under development.

GeologicConcept and GeologicRepresentation both have optional, many-to-many associations with Metadata, modeling the fact that any data object may have associated metadata and any Metadata object may be related to more than one data object. Currently, the only metadata classes in the model are Name and TextDescription. Thus, every GeologicConcept or GeologicRepresentation instance can have one or more names or free text descriptions. A name is an arbitrary text string used for human identification of an instance of a concept (data object). A preferred name may be specified for a concept within a GeologicVocabulary. The Name attribute preferred for a concept in any particular vocabulary provides a standard means of identifying the concept in that context. TextDescription is a narrative description of a data object to assist users in understanding the data. Other metadata classes, not explicitly modeled here, may relate to more than one instance; thus the need for a many-to-many relationship between Metadata and other concepts. 


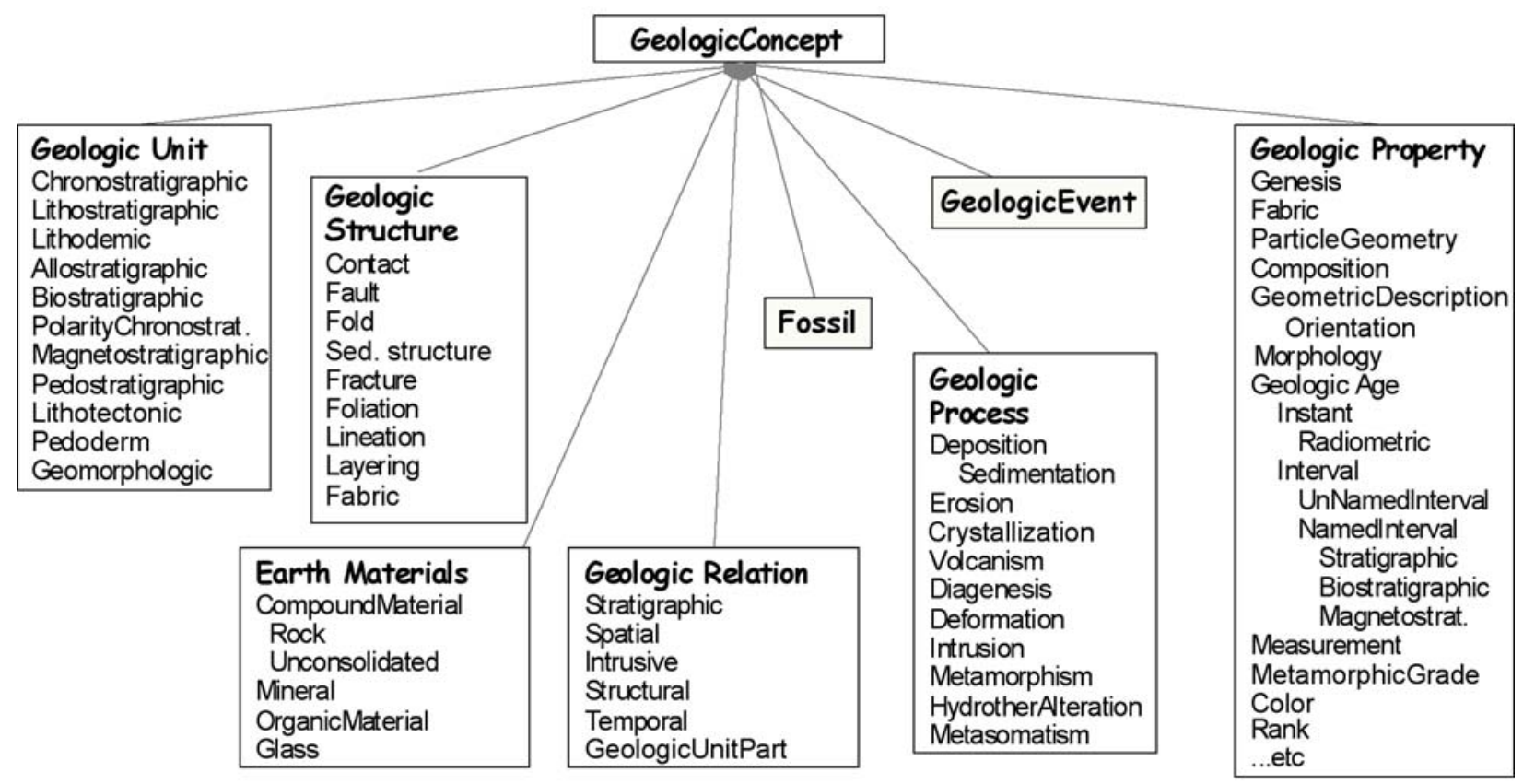

Figure 1. High-level geologic concept hierarchy.

Figure 1 is a generalized version of the modeled GeologicConcept hierarchy. The top-level concept that pertains to geologic maps and geologic information (loosely defined) is called Geologicconcept. The remaining boxes on Figure 1 represent additional concepts that inherit from GeologicConcept. In other words, GeologicStructure is a GeologicConcept and Contact is a GeologicStructure, and therefore a GeologicConcept.

Table 2. Major sub-concepts of GeologicConcept

\begin{tabular}{|l|l|}
\hline Concept & Scope and rationale \\
\hline EarthMaterial & $\begin{array}{l}\text { A naturally occurring substance in the Earth. EarthMaterial represents } \\
\text { substance, and is thus independent of quantity or location. Ideally, } \\
\text { EarthMaterials are defined strictly based on physical and chemical properties, } \\
\text { but because of traditional geologic usage, genetic interpretations enter into the } \\
\text { description as well. Does not include melted rock (magma or lava). Many concepts } \\
\text { related to water or petroleum have not been modeled in this version. }\end{array}$ \\
\hline Fossil & $\begin{array}{l}\text { The remains, trace, or imprint of a life form that has been preserved in an } \\
\text { EarthMaterial, and demonstrates evidence of having been changed from its } \\
\text { original biogenic form. Fossil is distinguished from biologic remains or biogenic } \\
\text { sedimentary structure based on evidence of having been converted incipiently or } \\
\text { substantially into a modified version of the original biogenic form or structure. } \\
\text { Although the passage of time is implicit in the definition of fossil, no constraint is } \\
\text { placed on the amount of time necessary to become a fossil. Includes ichnofossils, } \\
\text { casts and molds, as well as fossilized remains. Subtypes of fossil are also considered } \\
\text { kinds of CompoundMaterial to represent their material aspect or kinds of } \\
\text { Geologicstructure to represent their morphologic aspect. }\end{array}$ \\
\hline
\end{tabular}




\begin{tabular}{|c|c|}
\hline GeologicEvent & $\begin{array}{l}\text { An identifiable event during which one or more geologic processes act to modify } \\
\text { geologic entities. A GeologicEvent may have a specified GeologicAge and } \\
\text { GeologicEnvironment. An example might be a cratonic uplift event during } \\
\text { which erosion, sedimentation, and volcanism all take place. }\end{array}$ \\
\hline GeologicProcess & $\begin{array}{l}\text { A function, possibly complex, that acts on one geologic entity to produce another } \\
\text { geologic entity at a later time. Process is time independent; some } \\
\text { GeologicProcesses are observable in the present at work in the world or in the } \\
\text { laboratory, others can only be inferred from observing the results of the process. } \\
\text { Processes take one or more of EarthMaterial, GeologicUnit, or } \\
\text { GeologicStructure as input and have one or more of EarthMaterial, } \\
\text { GeologicUnit or Geologicstructure as output. }\end{array}$ \\
\hline GeologicProperty & An inherent feature used to characterize a GeologicConcept. \\
\hline GeologicRelation & $\begin{array}{l}\text { Any of a wide variety of relationships that can exist between two or more } \\
\text { GeologicConcepts. For example, the GeologicRelation 'intrudes' is a } \\
\text { relationship between an intrusive igneous rock and some host rock. Includes spatial, } \\
\text { temporal, sequence, correlation, and parent/child relations. Many of the relationships } \\
\text { in NADM-C1 (particularly attribute links and parent-child links) are not explicitly } \\
\text { modeled as kinds of GeologicRelation. }\end{array}$ \\
\hline GeologicStructure & $\begin{array}{l}\text { A configuration of matter in the Earth based on describable inhomogeneity, pattern, } \\
\text { or fracture. The identity of a geologic structure is independent of the material that is } \\
\text { the substrate for the structure. There are almost always strong dependencies between } \\
\text { the nature of the material substrate and the kinds of structure that may be present. A } \\
\text { disaggregated heap of particles does not have structure, and can only be described in } \\
\text { terms of the mineralogy and geometrical character of the constituent particles. } \\
\text { Geologic structures are more likely to be found in, and are more persistent in, } \\
\text { consolidated materials than in unconsolidated materials. Properties like 'clast- } \\
\text { supported', 'matrix-supported', and 'graded bed' that do not involve orientation are } \\
\text { considered kinds of Geologicstructure because they depend on the } \\
\text { configuration of parts of a rock body. Includes: sedimentary structures. }\end{array}$ \\
\hline GeologicUnit & $\begin{array}{l}\text { A body of earth material distinguished from adjoining material on the basis of } \\
\text { content (lithologic or fossil), inherent attributes, physical limits, geologic age, or } \\
\text { some other property or properties [adapted from NACSN, 1983, p.22; } \\
\text { http://www.agiweb.org/nacsn/code2.html]. Corresponds to 'stratigraphic unit' in the } \\
\text { North American Stratigraphic Code. Commonly used properties include } \\
\text { composition, texture, included fossils, magnetic signature, radioactivity, seismic } \\
\text { velocity, and age. Sufficient care is required in defining the boundaries of a unit to } \\
\text { enable others to distinguish the material body from those adjoining it [NACSN, } \\
1983 \text { ]. } \\
\text { A geologic unit is a part of the solid Earth that is identified by its geologic } \\
\text { characteristics, has definable, locatable boundaries, and is persistent in time. } \\
\text { Excludes non-material, temporal units. GeologicUnit instances are related using } \\
\text { the general GeologicRelation structure in NADM-C1. }\end{array}$ \\
\hline
\end{tabular}


Table 3. Major sub-concepts of GeologicRepresentation

\begin{tabular}{|c|c|}
\hline Concept & Scope and rationale \\
\hline GeologicClass & $\begin{array}{l}\text { A collection of occurrences on a geologic map or cross-section that represent a single } \\
\text { geologic concept and are depicted with the same symbol. The concept is typically a } \\
\text { geologic map unit or structure. In the case of a class representing a map unit, such as } \\
\text { the Dakota Sandstone, the collection includes all of the occurrences of the map unit } \\
\text { within the map area. The Spatialobject (line, polygon, etc.) associated with } \\
\text { each occurrence is depicted with the symbol associated with the GeologicClass. } \\
\text { The GeologicConcept associated with a GeologicClass is a summary } \\
\text { description of all the occurrences in the class. Each occurrence can also have a } \\
\text { unique description, and be assigned a unique symbol, either in addition to, or instead } \\
\text { of, the GeologicClass symbol. GeologicClass is distinct from } \\
\text { GeologicConcept because some concepts may be depicted using different } \\
\text { symbols on the same map. This allows symbolization based on criteria in addition to } \\
\text { the GeologicConcept. Examples: geologic contacts may be symbolized } \\
\text { differently based on location uncertainty; different bedding symbols are used for } \\
\text { horizontal, inclined, vertical and overturned, but all are representative of the } \\
\text { BeddingSurface concept. }\end{array}$ \\
\hline GeologicPortrayal & $\begin{array}{l}\text { A visualization designed to convey geologic information. GeologicPortrayal } \\
\text { includes GeologicMap, Crosssection, and Graphic. }\end{array}$ \\
\hline $\begin{array}{l}\text { GeologicPortrayal } \\
\text { Component }\end{array}$ & $\begin{array}{l}\text { Abstract super-class for concepts that are parts of the description of a geologic } \\
\text { portrayal. Includes MapLayout, MapDescription, Legend, LegendItem, } \\
\text { Geologicclass, Occurrence, and Spatialobject. }\end{array}$ \\
\hline GeologicVocabulary & $\begin{array}{l}\text { A collection of concept definitions, each associated with a preferred name, and } \\
\text { usually organized in some logical fashion such as in a hierarchy. The preferred name } \\
\text { associated with a concept in a GeologicVocabulary is a proxy for the collection } \\
\text { of property values and relationships specified in the definition. The vocabulary makes } \\
\text { the definitions of these concept instances available to apply in other descriptions } \\
\text { without having to reconstruct the entire description denoted by the concept definition. } \\
\text { Examples of geologic vocabulary include a collection of standard rock types, a } \\
\text { stratigraphic lexicon or a geologic time scale. }\end{array}$ \\
\hline Legend & $\begin{array}{l}\text { An ordered (potentially partial) collection of LegendItems. A map legend specifies } \\
\text { a collection of classified symbols (including patterns and colors) displayed on a } \\
\text { geologic map or cross-section, along with the meaning or geologic description of each } \\
\text { symbol. }\end{array}$ \\
\hline LegendItem & $\begin{array}{l}\text { An association of a GeologicClass or a Spatialobject with a symbol. Each } \\
\text { LegendItem instance represents a single entry in a map legend that describes either } \\
\text { a single entity (occurrence) or a single class of entities occurring on a geologic map or } \\
\text { cross-section. }\end{array}$ \\
\hline MapDescription & $\begin{array}{l}\text { All of the descriptive information that accompanies the graphic portion of a geologic } \\
\text { map or cross section. Includes descriptive text, symbols and their explanations, } \\
\text { associated graphics, etc. }\end{array}$ \\
\hline MapLayout & $\begin{array}{l}\text { A collection of geologic map, cross-section, and graphic elements, which together } \\
\text { constitute a complete geologic map. This concept roughly corresponds to an ESRI } \\
\text { ArcView } 3.2 \text { 'Layout' or ESRI ArcMap Document. }\end{array}$ \\
\hline
\end{tabular}




\begin{tabular}{|l|l|}
\hline Concept & Scope and rationale \\
\hline Occurrence & $\begin{array}{l}\text { An individual data item. Each occurrence differs from all other occurrences either in } \\
\text { some portion of its definition (GeologicConcept), or in its geometry (size, shape, } \\
\text { location). For example, two different outcrops of the same geologic unit (a Geo - } \\
\text { logicConcept) are different occurrences because they differ in geometry } \\
\text { (location). Similarly, the representations of the outcrop trace of the same fault (a } \\
\text { GeologicConcept) at different scales are different occurrences because their } \\
\text { geometric representation (Spatialobject) is different. Features which share the } \\
\text { same geometry, but different definitions, such as a fault and a contact that are } \\
\text { represented by the same line on a map, are also unique occurrences. }\end{array}$ \\
\hline SpatialObject & \begin{tabular}{l} 
A description of the geometry (size, shape, and location) of an occurrence. \\
\hline
\end{tabular} \\
\hline
\end{tabular}

\subsection{Role of geologic vocabularies}

Geologic vocabularies play a central role in the application of this model to store geologic information. Each vocabulary contains a collection of definitions. A definition is a normative description; it is associated with a preferred name in a vocabulary to become a controlled concept. The name associated with this concept implies the associated normative description. The normative descriptions may be simple text definitions, in which case the controlled concept is not analyzable. Each schema in this model that specifies a collection of properties, relationships and cardinalities associated with a geologic concept (plates 2, 3, 6, 7) defines a data structure (description schema) for describing instances of the concept. In an implementation of the model, an instance of one of these concepts is simply a data object that contains values for all properties and relationship required by the description schema. Such a concept instance may become a controlled concept if it is included in a geologic vocabulary, in which case the description becomes a normative description, or definition.

In the implementation of this model, the property values that populate descriptions of concept instances will be drawn from geologic vocabularies. A geologic vocabulary is a collection of concept definitions (ControlledConcept instances) that may then be used in the description of other concepts, particularly occurrence and Geologicclass instances included in a geologic map or cross-section legend. The descriptions of ControlledConcepts are normative-the controlled concept implies the associated description. Individual occurrences, or observations, may be described using the same description scheme, but the description applies only to a single instance and is referred to as an observation description. In an implementation of the model, there may be many concept descriptions in a data store; some of these may be observation descriptions, some may be normative descriptions. The structure of the descriptions is identical; the only distinction is inclusion of the description in a controlled vocabulary with some associated preferred name.

\section{DISCUSSION OF MODEL ELEMENTS (PLATES 1-13)}

Note that on the model diagrams some of the boxes represent simple attributes, while others may represent more complex structures that are defined more fully in other parts of the diagram. Some of these complex attributes have not been modeled in detail at this time. Notation on the diagrams follows standard UML conventions (see Rumbaugh and others, 1999).

\subsection{Top-Level Geologic Objects (Plate 1)}

The top-level elements of the data represent major concepts associated with the subset of the geologic domain considered herein. These elements are discussed in Section 3 (above) and depicted in the diagram below (Plate 1). 


\subsection{EarthMaterial (Plate 2)}

The substance of the solid Earth (rocks, minerals, organic material, glass...), is defined based on intrinsic properties independent of their disposition within the Earth. The identity of an Earth material is independent of extent (location or quantity). Magma, as an Earth material, is outside the scope of the model at this time.

EarthMaterial has properties of Color, Chemicaldescription (various sorts of chemical analyses), GeologicAge (Plate 4), PhysicalProperty (unspecified geotechnical or engineering properties) and EMGenesis (a subtype of Genesis, see Plate 7). EMGenesis represents a description of the history of the material's formation. An Earth material may be associated with one or more processes as either an input or product of the process. An Earth material may be the input to a variety of geologic processes that produce a variety of different Earth materials, and any given Earth material may be the product of a series of processes involving one or more starting Earth materials. Kinds of EarthMaterial include CompoundMaterial, Mineral, OrganicMaterial, InorganicFluid (including glass), and Void.

An Earth material that is an aggregation of constituents is a compound Earth material (CompoundMaterial in model). Two kinds of compound material are recognized, rock and unconsolidated material, which are distinguished based on their ConsolidationDegree property. Each constituent (EMConstituent) is an Earth material, possibly including other compound materials. Each constituent is described separately, including its role (EMCRole) in the compound material aggregation (e.g., phenocryst vs. groundmass, cement, and so on), the proportion of that constituent in the whole, and its relationship to other constituents (EMCRelation, such as overgrowth). Constituents may be specified based on their role in the compound material, allowing the same constituent material to be present in different roles, or constituents to be defined based on other criteria than their material. For example, clastic constituents, such as allochem, oolith, intraclast, pyroclast, autoclast, and epiclast, may be defined based on their genesis. The normative definition of 'pyroclastic rock' would be a Rock (a kind of CompoundMaterial), with 'igneous fragmentation' as its genesis, and only one constituent required--with role 'pyroclast', no material specified, and present in a proportion $>=75 \%$. A constituent may have a proportion of ' 0 ' to specify the absence of some constituent.

Relationships between constituents in a compound material can also be represented (EMCRelation), as well as the role each constituent plays in the relationship (EMCRelationRole). For example, in a relationship where albite forms overgrowths on K-feldspar phenocrysts, EMCRelation is 'overgrowth', the EMCRelationRole of albite in the relationship is 'encloses/overgrows', and the EMcRelationRole of K-feldspar is 'enclosed/overgrown'.

A compound material or a particular constituent in a compound material may be described by specifying ParticleGeometry and Fabric properties. ParticleGeometry represents description of particle size and particle shape, either qualitatively or quantitatively. Sorting terms are part of the qualitative description of particle size. Fabric is regarded as a type of geologic structure (Plate 6), focusing on the internal arrangement of the parts of the rock. Fabric is defined relative to an aggregation of particles considered as a unit. If the particles are disaggregated, the fabric disappears, but the particle geometry properties remain unchanged. Thus fabric includes concepts such as matrix-supported, clast-supported, and graded bedding, as well as metamorphic fabrics such as schistosity. Fabric or particle geometry may be described for a particular constituent, or for the entire aggregation of constituents in a compound material. The description for the constituents must be consistent with the description for the whole aggregation. The logic of this consistency check is complex, and probably requires intervention by a geologist to enforce.

Void represents volume in an Earth material that is either empty or filled with a fluid (gas or liquid). This concept is included as a kind of EarthMaterial to allow description of gas bubbles, porosity, and fluid inclusions using the constituent aggregation description scheme. Porosity is expressed as the proportion of a void constituent in a compound material. The geometry and connectivity of void constituents are described using ParticleGeometry properties. The details of such description have not been modeled. 


\title{
4.3 GeologicUnit (Plate 3)
}

GeologicUnit represents an identifiable part of the Earth. A geologic unit is a volumetric body of Earth material distinguished by content (lithologic or fossil), inherent attributes, physical limits, or geologic age [North American Code of Stratigraphic Nomenclature (NACSN), 1983]. There are a number of subtypes of GeologicUnit. Most are defined in the North American Stratigraphic Code (NACSN); a few additional subtypes were defined for NADM-C1.

\section{From NACSN:}

\author{
Allostratigraphic \\ Biostratigraphic \\ Chronostratigraphic \\ Lithodemic \\ Lithostratigraphic \\ Magnetostratigraphic \\ Pedostratigraphic \\ Polarity-Chronostratigraphic \\ Additional unit types: \\ Geomorphologic - a unit defined by surface morphology \\ Lithotectonic - a unit defined by geologic history or deformation \\ Pedoderm - a mappable surface-soil unit (as defined in Jackson (1997)
}

The history of a geologic unit is described by the GUGenesis property (a subtype of Genesis (see Plate 7)). As is the case with Earth materials, a geologic unit may be associated with one or more geologic processes as either input or product.

A Geologicunit is composed of GeologicUnitParts in a manner analogous to the composition of a compound material by Earth material constituents. Each geologic unit part has a role (GURole) and a proportion in the whole unit, and may have relationships with other parts of the geologic unit (GUPRelation, GUPRelationRole). For example, in a relationship where one part of a geologic unit overlies another, GUPRelation is 'overlies', the overlying GeologicUnitPart has the GUPRelationRole 'UpperPart', and the overlain GeologicUnitPart has the GUPRelationRole 'LowerPart'. A geologic unit part is either a compound material or another geologic unit (e.g. member of a formation). The diagram in the model (Plate 3) does not use standard UML notation for this subtype relationship because of limitations in the modeling software used to draw the plates. The relationships are drawn as standard associations with a stereotype of '«IsA»'.

Properties of geologic units are descriptive of the whole body of material represented. These include color, extent (the spatial distribution of the unit), geologic age, metamorphic grade, morphology (the geometry or form of a rock body), outcrop character, rank, and weathering character. Most of these properties (except rank) could be modeled as complex attributes. Geologic age is the only property currently modeled in more detail (see Plate 4).

\subsection{GeologicAge (Plate 4)}

GeologicAge represents the specification of the age (time before present) associated with some geologic event, measured in years. A geologic age may be based on various geochronologic methods, by correlation with some material referent, or expressed in relative terms. A GeologicAge may be an AgeDate, which represents a geologic 'instant', or an AgeInterval, which represents some interval of time. UnnamedAge in NADM-C1 corresponds to the Geochronometric unit of the North American Stratigraphic Code [NACSN, 1983], and NamedAge corresponds to the Geochronologic unit of the NACSN. We have chosen to use different names in order to avoid confusion between unit as (1) an interval of time and (2) a body of rock. 


\title{
4.5 Fossil (Plate 5)
}

Fossils are sometimes recognized by the form that is imprinted in an Earth material, and sometimes recognized as the preserved remains of a life form. Fossils are typically thought of as objects (c.f. Jackson, 1997: "Said of any object...”), whereas EarthMaterial represents substance. Geoscientists commonly describe fossils as constituents of rocks.

The model distinguishes fossils that are tangible compound materials (e.g., Cast and PreservedRemain) from those that are geologic structures (e.g., Molds and TraceFossil). In future versions of the model, these may need to be distinguished with different names (e.g. FossilMaterial, and Fossilstructure) because of the differences in how they are used in rock description, and in how they are described. The diagram allows the proportion of fossils in a compound material to be described explicitly, because the constituents of a compound material may be other compound material, and Fossil as a material is a kind of CompoundMaterial. The mineral composition and structure of a fossil can also be described explicitly in the same way that the mineral composition and structure of a rock (or any other compound material) can be described.

\subsection{GeologicStructure (Plate 6)}

Geologicstructure represents the configuration of a specific portion of the Earth's material. Geologic structure in a purely descriptive sense is based on describable inhomogeneity, pattern or discontinuity in a rock mass. The scale of geologic structures ranges from microscopic (micron-scale) to megascopic (km-scale). Perception of geologic structure depends on detectable inhomogeneity in a rock body. Examples of such inhomogeneities include fractures, mineral grain boundaries, and boundaries between parts of the rock with different particle geometry (texture) or composition. Geologic structure is grounded in relationships between parts of a rock or rock body. As used here, it includes sedimentary structures.

The identity of a geologic structure is independent of the material that is the substrate for the structure. There are almost always strong dependencies between the nature of the material substrate and the kinds of structure that may be present. A disaggregated heap of particles does not have structure, and can only be described in terms of the mineralogy and geometrical character of the constituent particles. Geologic structures are more likely to be found in, and are more persistent in, consolidated materials than in unconsolidated materials. Geologic structures may be present in outcrops (deposits) of an unconsolidated material.

Specific types of geologic structures defined include:

\author{
Contact \\ Fabric \\ Fault (with attribute Displacement) \\ Fold \\ Foliation \\ Fracture (example Joint) \\ Layering \\ Lineation \\ PreLithificationStructure (Used for both sedimentary and igneous rocks. \\ Example: BeddingSurface)
}

A geologic structure may be compound if it is composed of other geologic structures. In the model, CompoundGeologicstructure is an aggregation of one or more Geologicstructure instances. CompoundFabric is a type of CompoundGeologicStructure. An example of a compound fabric would be a foliation that contains a lineation.

The properties of geologic structure in the model include genesis, age, geometry, defining elements, and pervasiveness. All aspects of orientation, thickness, planar vs. linear geometry, fold morphology, etc. that may be used to describe a geologic structure are encompassed in the GeometricDescription property. This must 
obviously be a complex property, but geometric description has not been modeled in detail. Pervasiveness specifies the degree to which a structure is continuous throughout a body of rock, considered at some scale of reference. The defining elements (GSDefiningElement property) specify the nature of inhomogeneity in a rock body that defines a geologic structure. Examples include Discontinuity, ShapedSurface, orientedParticle, MaterialBoundary, and Layer.

After several extended discussions attempting to classify geologic structures in a hierarchical way (e.g., penetrative vs. discrete; planar vs. linear; rock forming vs. rock modifying), the committee settled on using a relatively flat, non-hierarchical representation in the model. Multiple valid hierarchies for geologic structures are possible depending on the attributes chosen to guide branching at each level in the hierarchy; each is suited for different purposes. The team opted for a flat structure that does not commit to any one hierarchy.

\title{
4.7 GeologicProcess and Genesis (Plate 7)
}

A geologic process is a function, possibly complex, that acts on one geologic entity to produce another geologic entity. Geologic processes are time independent; some processes are observable in the present at work in the world or in the laboratory, others can only be inferred from observing the results of the process. GeologicProcess types in NADM-C1 include the following:

\author{
Crystallization \\ Deformation \\ Deposition (Example: Sedimentation) \\ Diagenesis \\ Erosion \\ HydrothermalAlteration \\ Intrusion \\ Metamorphism \\ Metasomatism \\ Volcanism
}

A GeologicProcess may be associated with a GeologicEvent as part of a Genesis. A GeologicProcess may have one or more instances of EarthMaterial, Geologicunit, or GeologicStructure associated as input and one or more instances of EarthMaterial, Geologicunit or GeologicStructure associated as a resultant product.

A geologic history (Genesis) is modeled as an ordered aggregation of geologic events (GeologicEvent). A geologic history may apply to an Earth material, a geologic unit, or a geologic structure. Geologic events may be at least partially overlapping, even though the model specifies that the GeologicEvent instances that constitute a Genesis are ordered. The data model design team recognizes that geologic events are typically continuous rather than discrete (instantaneous) but suspect that this arrangement will suffice for most uses. GeologicEvent may be inadequate for use in simulation. Each geologic event may have an age (GeologicAge), a geologic environment, and one or more associated geologic processes. Geologic environment is not modeled in detail, but is construed broadly to include physical settings on the earth surface specified by climate, tectonics, physiography or geography, and settings in the Earth's interior specified by pressure, temperature, chemical environment, or tectonics.

\subsection{GeologicRelation (Plate 8)}

This concept represents a general mechanism for expressing relationships between any geologic concepts. The model recognizes several specific types of relationships for illustrative purposes:

IntrusiveRelation

SpatialRelation

StratigraphicRelation 
StructuralRelation

TemporalRelation

Two or more geologic concepts are associated in a GeologicRelation; each has a GeologicRole in the relationship. Examples of geologic roles include "overlies", "is overlain by", "is younger", "is older", "intrudes", "is intruded by", and so forth. In a relationship where an igneous unit intrudes a sedimentary unit, the geologic relationship is 'intrudes', the intruded sedimentary unit has the role 'host', and the igneous unit has the role 'intrusion'. Most of the information on a geologic map could be described using this general relationship mechanism, but we have explicitly represented several geologic relationships in the model to emphasize their significance. Many other types of relationships can also be accommodated via GeologicRelation, for example, topologic relations between spatial objects could be described where they are scientifically significant.

GeologicProperty (Plate 9) and (Plate 10)

GeologicProperty represents concepts that are properties (attributes) of geologic concepts. These property concepts are connected to other concepts through relations and appropriate roles. Many geologic property values are specified by terminology, and in practice will have an associated GeologicVocabulary of values (instances) produced by the NADM Science Language Technical Team or other users.

\subsection{GeologicVocabulary (Plate 11)}

A GeologicVocabulary is a collection of terms (generally science language terms) and their associated definitions (GeologicConcept), usually organized in some logical fashion such as in a hierarchy. Typical examples of geologic vocabularies are individual rock classification schemes (collections of Earthmaterial instances), time scales (collections of GeologicAge instances), and other classifications or taxonomies. Constraints on the completeness or uniqueness of the concepts in a vocabulary relative to the domain from which they are drawn are not explicitly modeled. A geologic vocabulary may describe a formal standard or any individual's [self-consistent] usage of terms; it is not restricted to formally published vocabularies. A geologic vocabulary may also represent a pick list of terms for use in terminological description. The most useful classification schemes/lists are hierarchical.

Although many names may be associated with a geologic concept (through the Metadata association, Plate 1); one name is designated as preferred within a particular vocabulary in order to facilitate communication.

\section{GeologicPortrayal (Plate 12) and GeologicPortrayalComponent (Plate 13)}

GeologicPortrayal represents a visualization of spatially referenced geologic information. Individual visualizations of data from the database are designed by a geologist to communicate a particular understanding of the geology, and the choice of what to symbolize and how it is symbolized has a significant impact on how the map communicates that understanding to a user. Different visualizations based on the same data might communicate different aspects of the geology.

GeologicPortrayal is subtyped into CrossSection, GeologicMap, and Graphic. Cross-sections and geologic maps are graphical depictions in which the spatial elements have some mathematical correspondence with location on (or in) the Earth. A graphic is a figure designed to convey information visually in which the arrangement of graphic elements is not a mapping (in the geodetic sense) to a location on/in the Earth, but may follow some other logic. Correlation charts, explanations of map units, ternary diagrams to show composition, and text blocks are examples of graphics.

One or more GeologicPortrayal instances may be included in a MapLayout, which represents the collection of graphical elements that are assembled to produce a complete geologic map along with the arrangement of those elements (not modeled explicitly in this version). A full map layout may include the map itself, cross-sections, a legend, accompanying text and figures, correlation diagram(s), and/or stratigraphic column(s). 
represents an atomic graphic element, which may not be separated into meaningful parts. Symbols are associated with a geologic class in a map legend, or are associated with spatialobject instances to symbolize particular geologic occurrences.

Each GeologicMap or CrossSection instance has one associated MapDescription, which specifies the geologic horizon, scale, and projection of the visualization. A cross-section is considered a special kind of geologic map in a vertical plane. Individual MapDescription instances are either associated with exactly one GeologicMap instance or exactly one CrossSection instance. The map description also specifies a base map (BaseMap), which is an associated visualization that provides a viewer with reference to known cultural and physiographic features. Scale and projection properties determine the mathematical relationship between locations in the visualization and locations in or on the Earth. Horizon refers to the surface on which features are mapped, commonly surface topography, but also may be a vertical surface (for a cross-section), a horizontal surface (mine level), an unconformity, a fault surface (fault cut-off map), etc. In 3-D systems, the horizon will be a volume. Most lines (Spatialobject) on a geologic map represent the intersection of 3-D geologic surfaces with a map horizon. A base map typically depicts a single horizon, but geologic lines related to different horizons may be displayed on a single map. For example, structure contours represent the intersection of a geologic surface with a series of horizons, each one a horizontal surface at a particular elevation, all of which are probably different from the horizon of the base map on which they are displayed.

A MapDescription consists of a collection of Legend and 0ccurSymbol (occurrence symbolization) instances. These specify the Spatialobject instances to display, the symbols used to depict them, and the connections (GeologicClass) between the spatial objects and the meaning of the symbols (GeologicConcept). Spatialobject represents the spatial entities (points, lines, areas, surfaces, volumes) depicted on the map. The Legend is an ordered collection of LegendItem instances, which define a collection of symbols, each associated with a specific geologic concept (via ClassSymbol and GeologicClass) or a specific Spatialobject instance (via OccurSymbol). The Legend specifies the points, lines and polygons (Spatialobject) that are displayed, either directly through the 0ccurSymbol relationships, or indirectly through GeologicClass (via Occurrence). The notion here is that symbolization may be assigned to classes (i.e. all occurrences on the map for the Geologicunit "Idaho batholith", or all normal faults), while allowing for individual exceptions (i.e. a certain pluton may be symbolized differently). As another example, the mines on the map may all be assigned the same symbol (through the GeologicClass relationship), but one particular mine of special interest may be given a unique symbol (through the occursymbol relationship). Classsymbol therefore represents the symbol for a specific class of objects on the map. 0ccurSymbol associates a Symbol with a particular Spatialobject, with the rule being that occurSymbol assignments take precedence over Classsymbol assignments. Occurrence-symbol relationships may also be used to denote specific cartographic characteristics such as the rotation angle for a symbol, e.g. structural measurements.

Geologicclass represents the collection of objects shown on a map associated with a specific geologic concept. Each GeologicClass is associated with exactly one GeologicConcept that assigns its meaning. A geologic concept may be associated with multiple geologic classes, but in any one MapDescription the association is unique in both directions.

Spatialobject represents a geometric entity such as a point, line, area, or volume-note that these subtypes of Spatialobject are not specified, allowing for 2-D or 3-D variations. Each Spatialobject is associated with projection, scale, and horizon properties. The horizon object defines the surface on which the feature is mapped [possibly a volume in 3-dimensional models].

Acknowledgements. The DMDT would like to thank the many people and organizations who contributed time and support to the development of NADM-C1. The DMDT would also like to acknowledge the NADM Science Language Technical Team for their productive interactions and useful materials, the reviewers (Jon Matti, Peter Davenport) for carefully reviewing this manuscript and the model, and the American Geological Institute for graciously permitting direct and indirect re-use of select definitions from the AGI Glossary ( $4^{\text {th }}$ Edition, Jackson 1997). 


\section{REFERENCES CITED}

Allen, 1970, Towards a general theory of actions and time. Artificial Intelligence v. 23, p. 123-154.

American Commission on Stratigraphic Nomenclature, 1970, Code of Stratigraphic Nomenclature ( $2^{\text {nd }}$ ed.): American Association of Petroleum Geologists, Tulsa, Okla., 45 p.

Brewer, R., Crook, K. A. W. and Speight, J. A., 1970, Proposal for soil stratigraphic units in the Australian stratigraphic code. J. Geol. Soc. Aust., v. 17, p. 103-111.

Brodaric, Boyan, and Gahegan, M., 2000, Geoscience Map Data Models, Open Systems GIS and Semantics, in Proceedings, GeoCanada2000-The Millenium Geoscience Summit, Calgary, Alberta, p. 7.

Brodaric, Boyan, and Hastings, Jordan, 2002, An Object Model for Geologic Map Information, in Proceedings of the Spatial Data Handling 2002 Symposium, Ottawa, Canada.

Brodaric, B., Journeay, M., Talwar, S., and Boisvert, E., 1999, Cordlink Digital Library. Geological Map Data Model, Version 5.2, June 18, 1999, accessed at http://cordlink.gsc.nrcan.gc.ca/cordlink1/info_pages/English/dm52.pdf.

Davis, G. H., 1984, Structural Geology of Rocks and Regions: New York, John Wiley and Sons, 492 p.

DMDT, 2002, Progress Report: North American Geologic Map Data Model (NADM) Design Team, in Soller, D. R., Ed., Digital Mapping Techniques 2002, Workshop Proceedings, U.S. Geological Survey Open-File Report 02-370, p. 4146, accessed at http://pubs.usgs.gov/of/2002/of02-370/dmdt.html.

DOLCE, accessed at http://www.loa-cnr.it/DOLCE.html.

Gangemi A., Guarino N., Masolo C., Oltramari, A., Schneider L., 2002, Sweetening Ontologies with DOLCE: Proceedings of EKAW 2002, Siguenza, Spain, accessed at http://www.isib.cnr.it/infor/ontology/Papers/DOLCE-EKAW.pdf.

Hansen, Edward, 1971, Strain facies: New York, Springer-Verlag, 207 p.

Hobbs, B.E., Means, W.D., and Williams, P.F., 1976, An Outline of Structural Geology: New York, John Wiley and Sons, $571 \mathrm{p}$.

Jackson, J.A., 1997, Glossary of geology, 4th ed.: Alexandria, Virginia, American Geological Institute, 769 p.

Johnson, B. R., Brodaric, Boyan, Raines, G. L., Hastings, J. T., and Wahl, Ron, 1999, Digital Geologic Map Data Model, version 4.3: AASG/USGS Data Model Working Group Report, accessed at http://geology.usgs.gov/dm/model/Model43a.pdf.

Lenat, D. B., 1995, Cyc: A Large-Scale Investment in Knowledge Infrastructure: The Communications of the ACM, v.. 38, p. 33-38.

Means, W.D., 1976, Stress and Strain: New York, Springer-Verlag, 339 p.

North American Commission on Stratigraphic Nomenclature (NACSN), 1983, North American stratigraphic code: American Association of Petroleum Geologists Bulletin, v. 67, no. 5, p. 841-875, accessed at http://www.agiweb.org/nacsn/code2.html.

OpenCyc, accessed 11/12/03 at http://www.opencyc.org.

Parsons, R. B., 1981, Proposed soil-stratigraphic guide, in International Union for Quaternary Research and International Society of Soil Science: INQUA Commission 6 and ISSS Commission 5 Working Group, Pedology, Report, p. 612.

Passchier, C.W., and Trouw, R.A.J., 1998, Microtectonics: $2^{\text {nd }}$ corrected reprint: New York, Springer-Verlag, 289 p.

Pease, A., Niles, I., 2002, IEEE Standard Upper Ontology: A Progress Report: Knowledge Engineering Review, Special Issue on Ontologies and Agents, v. 17.

Ramsay, J.G., 1967, Folding and Fracturing of Rocks: New York, McGraw-Hill, 568 p.

Richard, S. M., 1999, Geologic concept modeling, with examples for lithology and some other basic geoscience features, in Soller, D. R., Ed., Digital Mapping Techniques 1999, Workshop Proceedings, U.S. Geological Survey Open-File Report 99-386, p. 59-75, accessed at http://pubs.usgs.gov/of/of99-386/richard.html.

Richard, S. M., submitted, Geoscience Concept Models: submitted, K. Sinha and C. Baru, eds., Geoinformatics, Geol. Soc. America Special Paper.

Richard, S.M. and Orr, T.R., 2001, Data structure for the Arizona Geological Survey geologic information system; basic geologic map data, in Soller, D. R., Ed., Digital Mapping Techniques 2001, Workshop Proceedings, U.S. Geological Survey Open-File Report 01-223, p. 167-188, accessed at http://pubs.usgs.gov/of/of01-223/richard2.html.

Robertson, S., 1999, BGS Rock Classification Scheme, Volume 2, Classification of metamorphic rocks: British Geological Survey, Research Report RR 99-02, 24 p.

Rumbaugh, J., Jacobson, I., Booch, G., 1999, The unified modeling language reference manual: Reading, MA, AddisonWesley. 
Soller, D. R., Brodaric, Boyan, Hastings, J. T., Wahl, Ron, and Weisenfluh, G. A., 2002, The Central Kentucky Prototype: An object-oriented geologic map data model for the National Geologic Map Database: U.S. Geological Survey Open-File Report 02-202, 39 p., accessed at http://pubs.usgs.gov/of/2002/of02-202/of02-202.pdf.

Sowa, J. F., 2000, Knowledge Representation: Logical, Philosophical, and Computational Foundations: Pacific Grove, CA, Brooks Cole Publishing Co., 594 p.

Stanford, L.R., Othberg, K.L., Lewis, R.S., and Breckenridge, R.M., 1999, A digital geologic map data model designed for GIS users, in Soller, D. R., Ed., Digital Mapping Techniques 1999, Workshop Proceedings, U. S. Geological Survey Open-File Report 99-386, p. 169, accessed at http://pubs.usgs.gov/of/of99-386/stanford.html.

Stanford, L.R., 2002, A Brief Documentation of the Idaho Geological Survey’s Digital Geologic Map Data Model, Version 2.1: A Variant of the North American Digital Geologic Map Data Model, Version 4.3, accessed at http://www.idahogeology.com/Lab/pdf_files/DataModel/IGS-model-v2.1.pdf.

SUMO ontology, accessed 11/12/03, http://ontology.teknowledge.com/.

Turner, F.J., and Weiss, L.E., 1963, Structural Analysis of Metamorphic Tectonites: New York, McGraw-Hill, 545 p.

Walker, P. H., Beckmann, G. G. and Brewer, R., 1984, Definition and use of the term 'pedoderm'. J. Soil Science, v. 35, p. 505-510.

Webster’s encyclopedic unabridged dictionary of the English Language: Merriam-Webster Inc. 


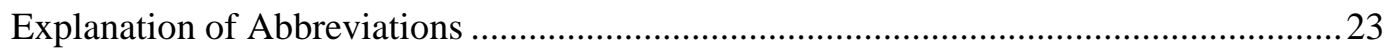

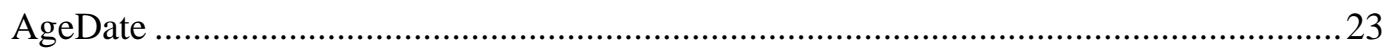

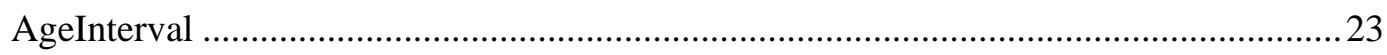

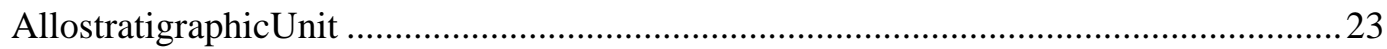

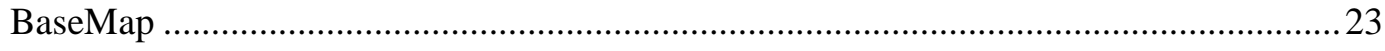

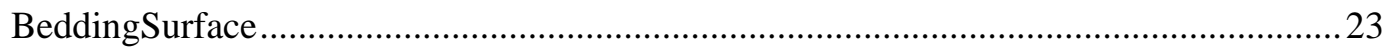

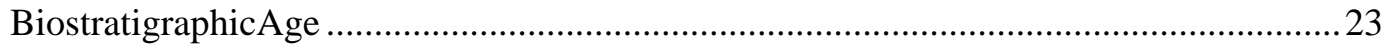

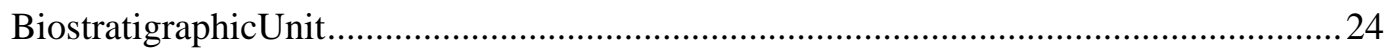

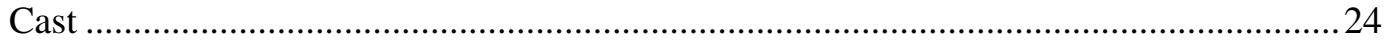

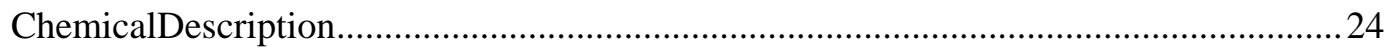

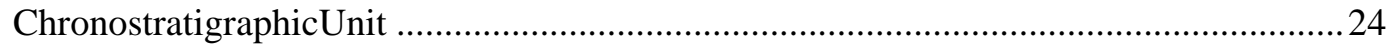

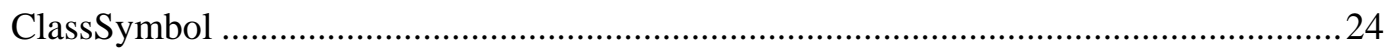

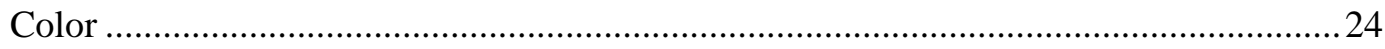

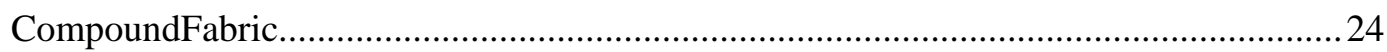

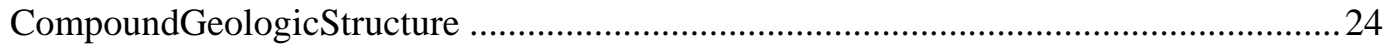

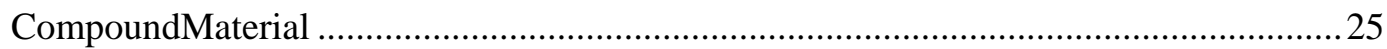

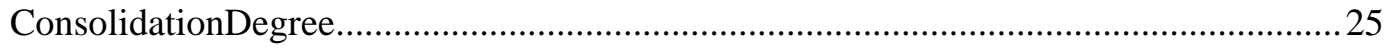

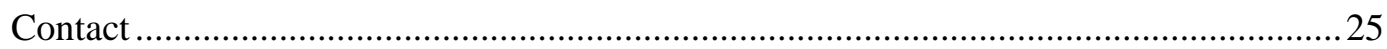

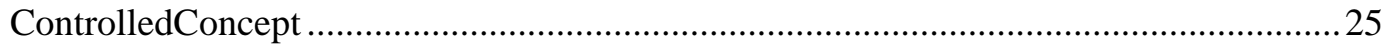

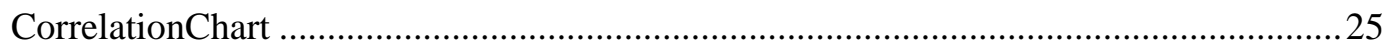

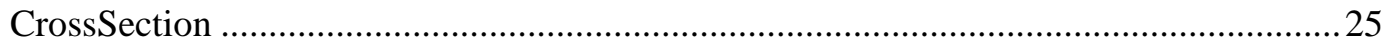

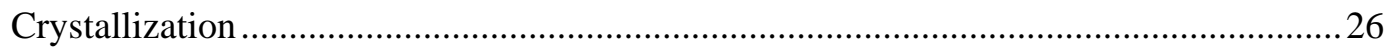

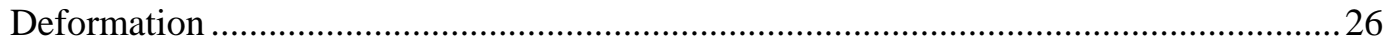

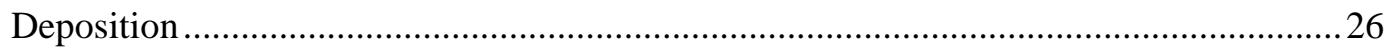

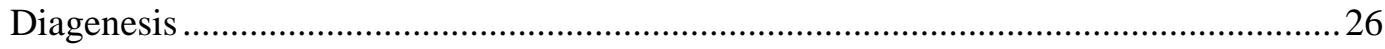

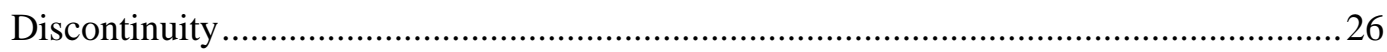

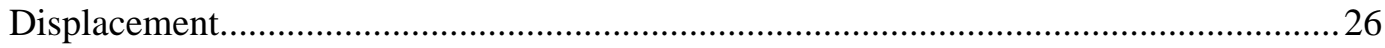

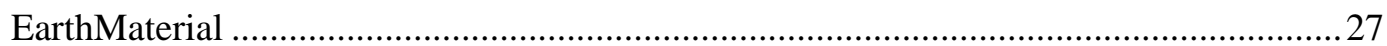

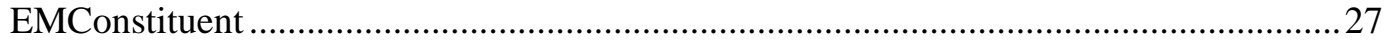

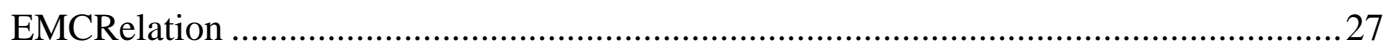

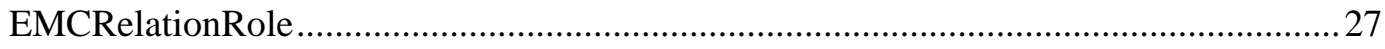

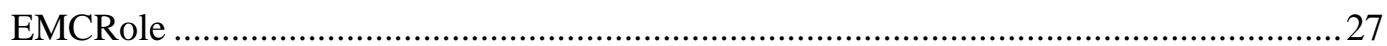

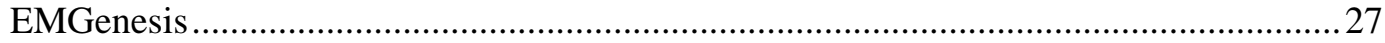

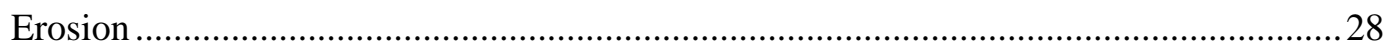

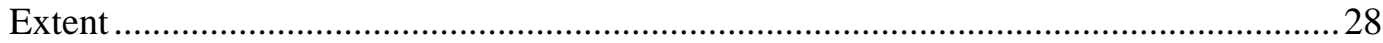

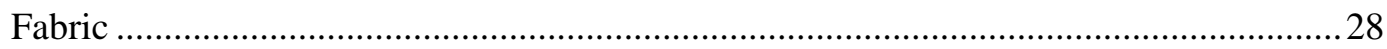

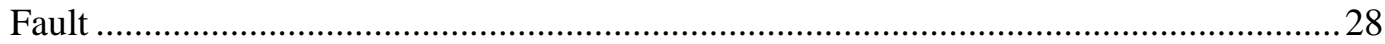

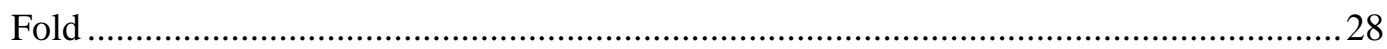

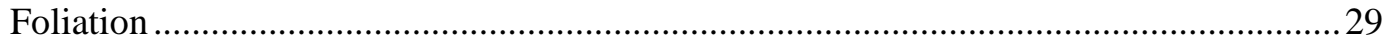

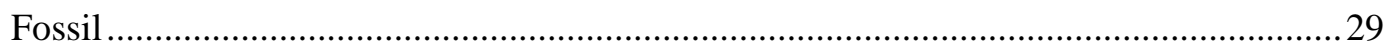




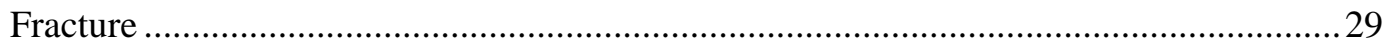

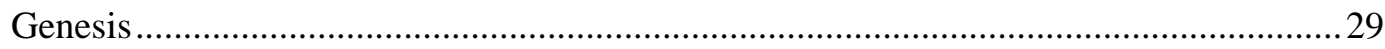

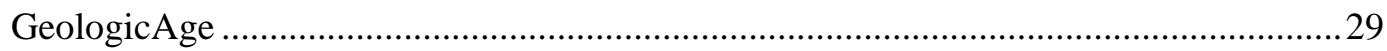

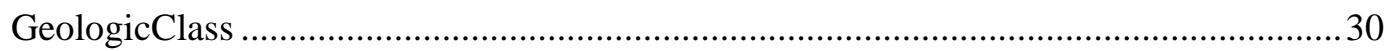

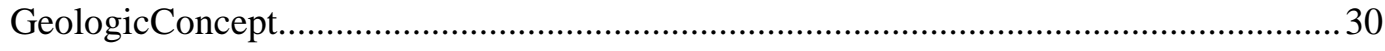

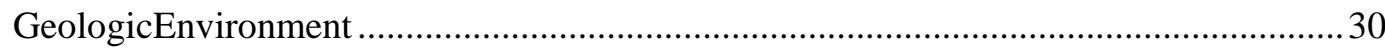

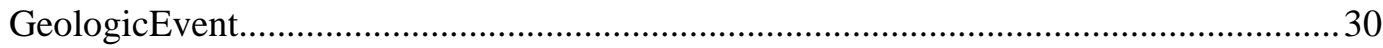

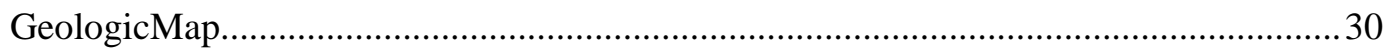

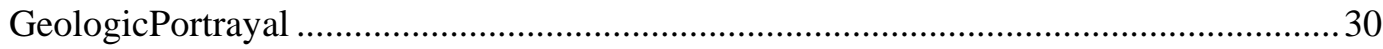

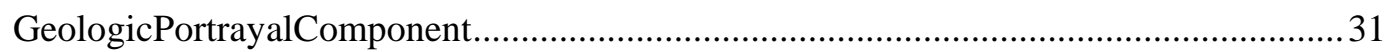

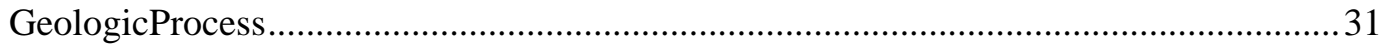

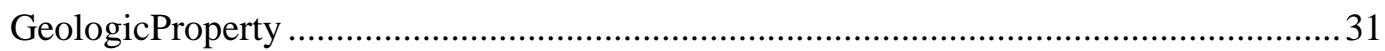

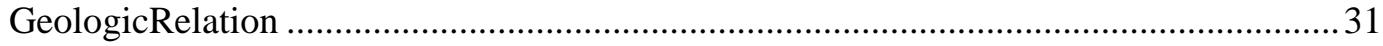

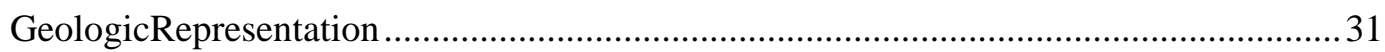

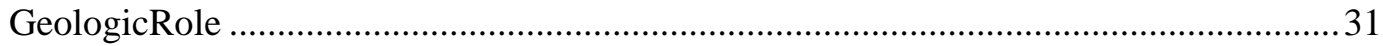

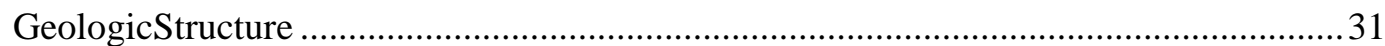

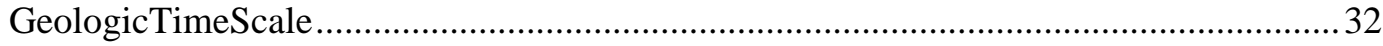

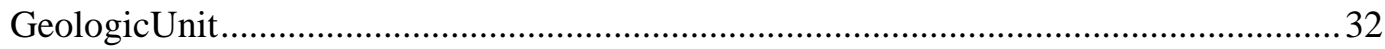

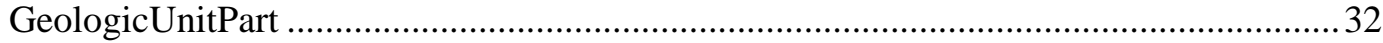

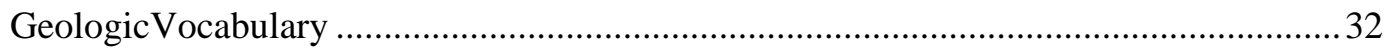

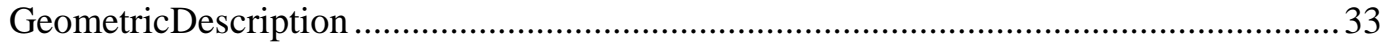

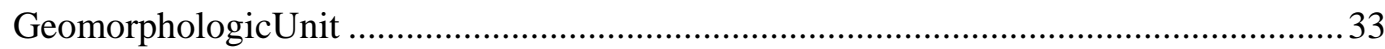

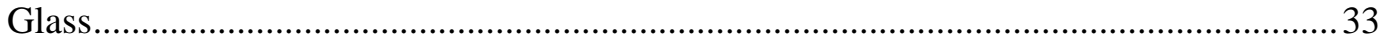

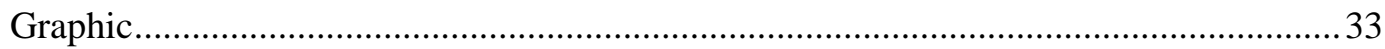

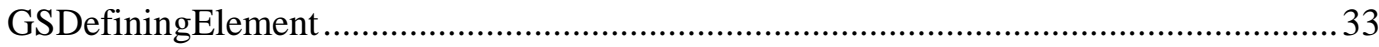

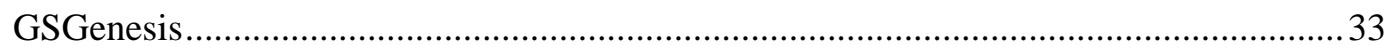

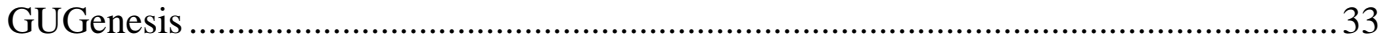

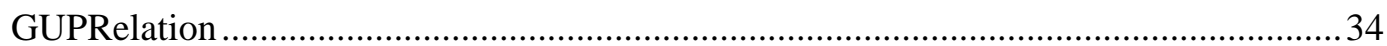

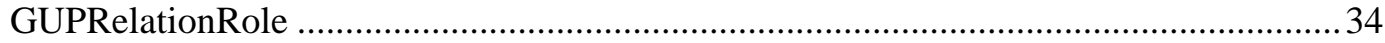

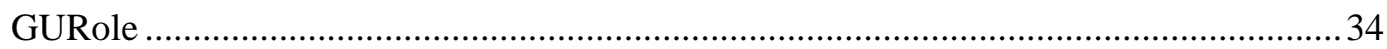

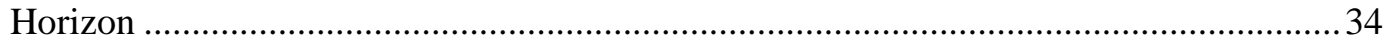

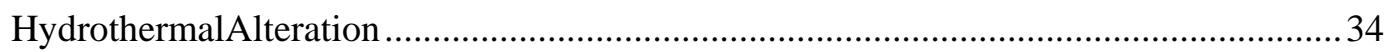

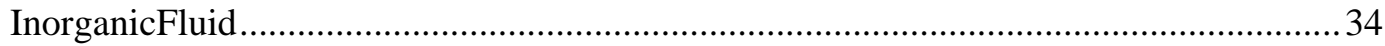

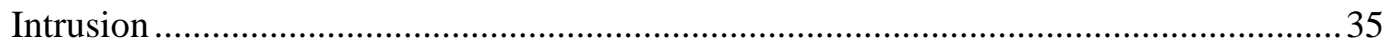

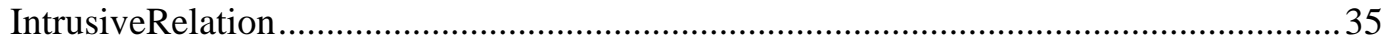

Joint

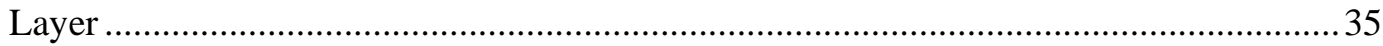

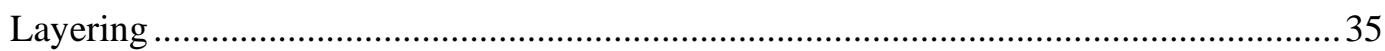

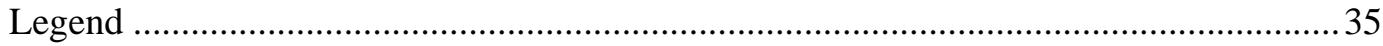

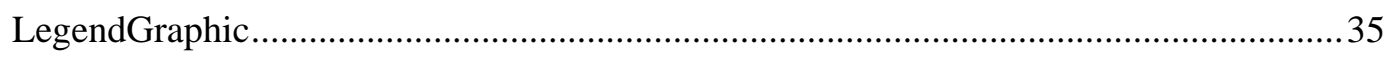

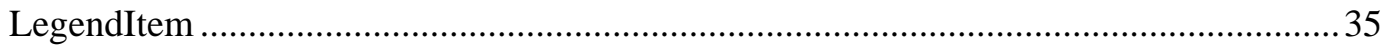




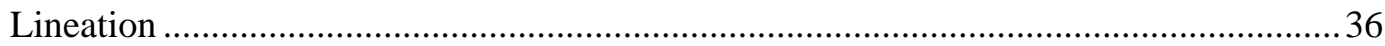

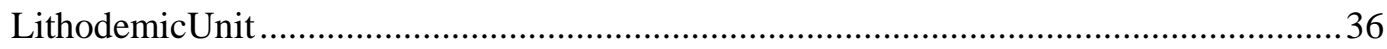

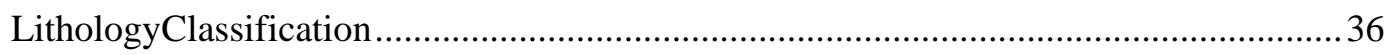

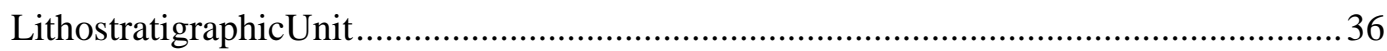

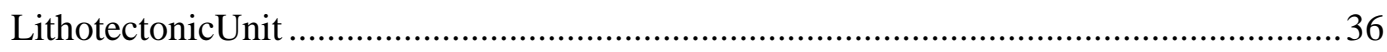

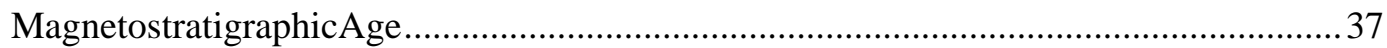

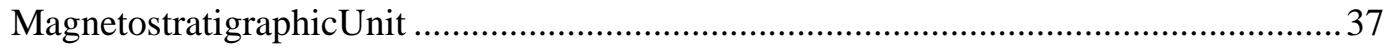

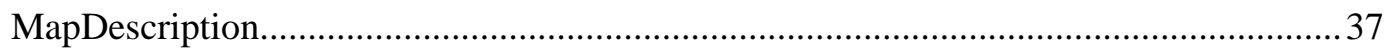

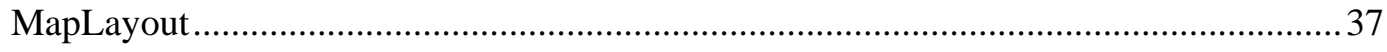

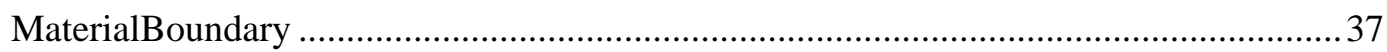

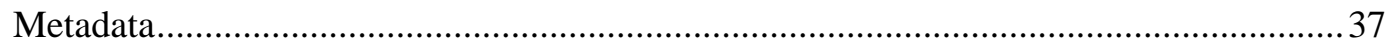

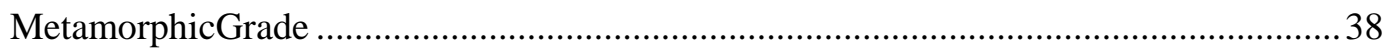

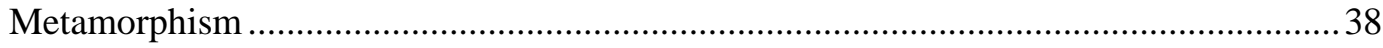

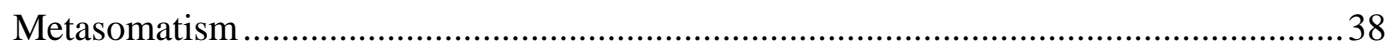

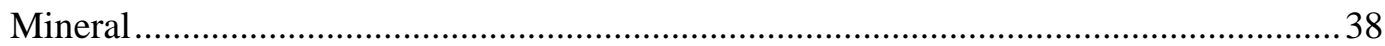

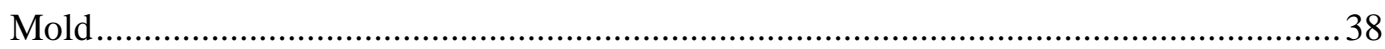

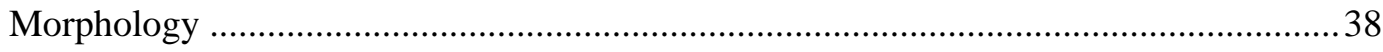

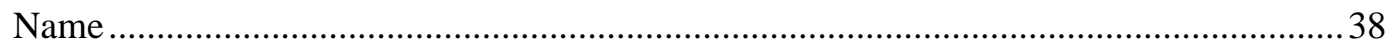

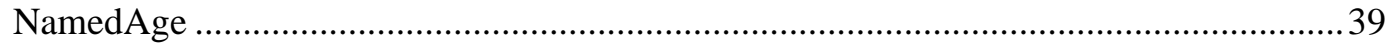

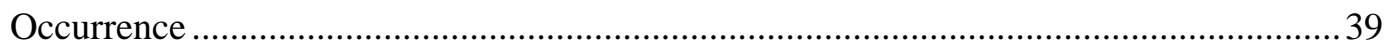

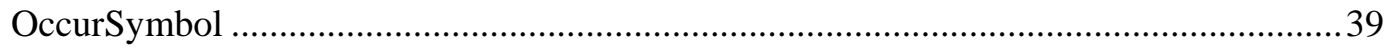

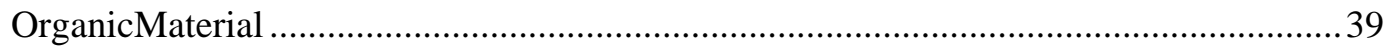

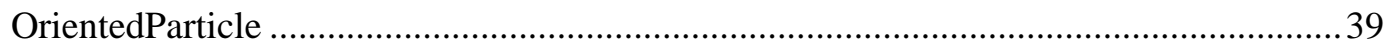

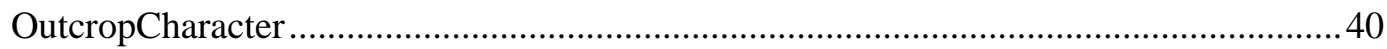

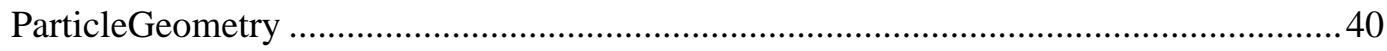

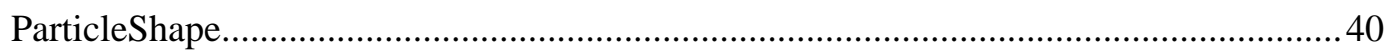

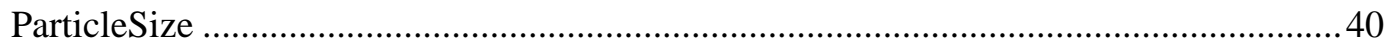

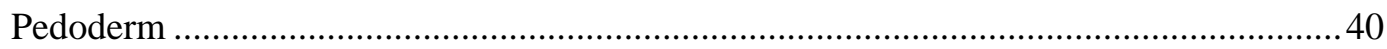

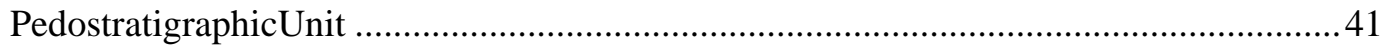

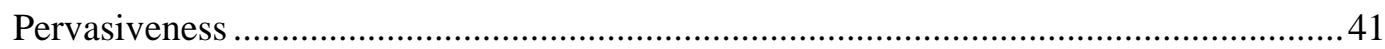

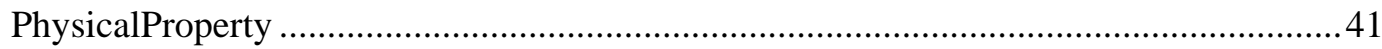

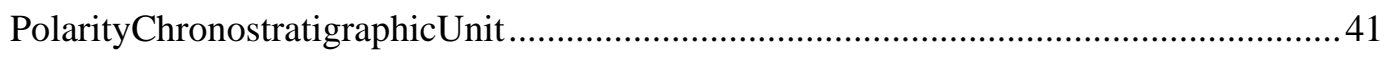

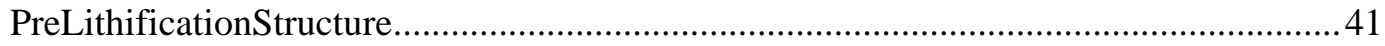

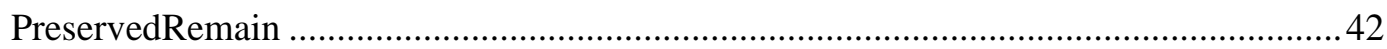

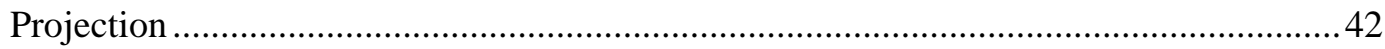

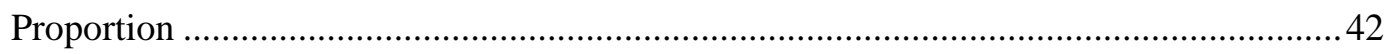

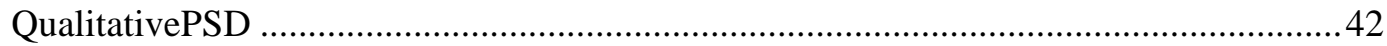

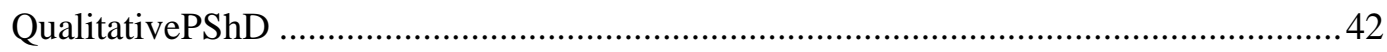

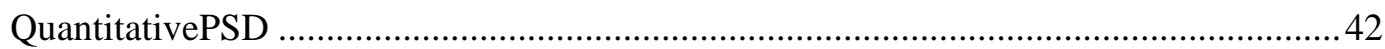

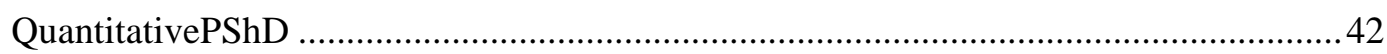

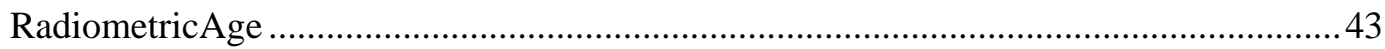




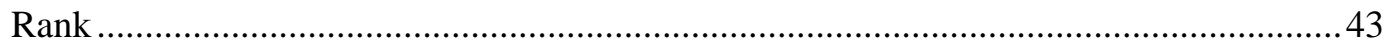

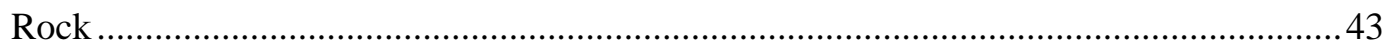

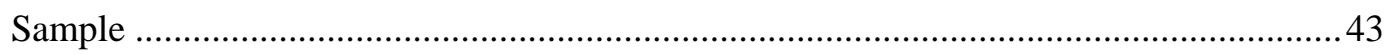

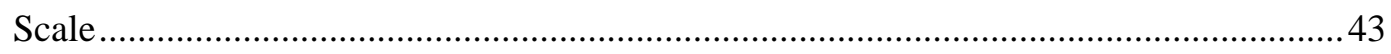

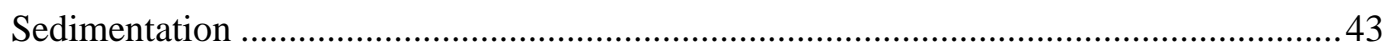

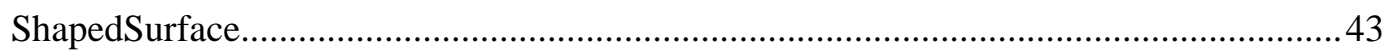

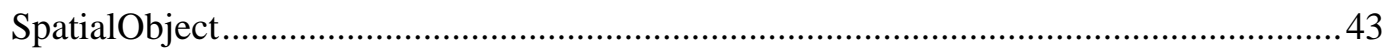

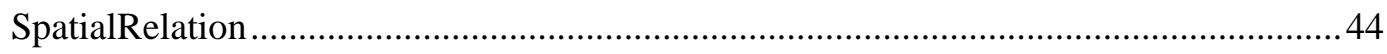

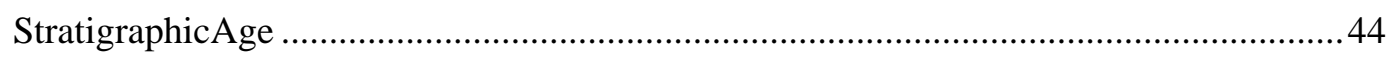

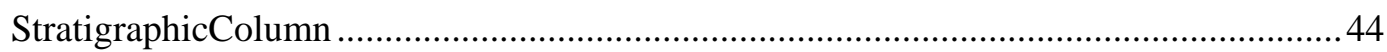

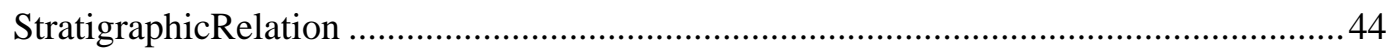

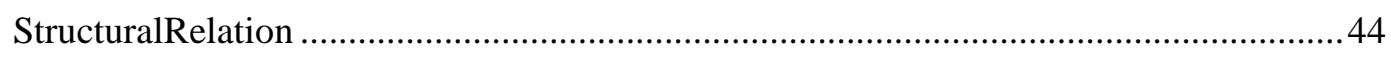

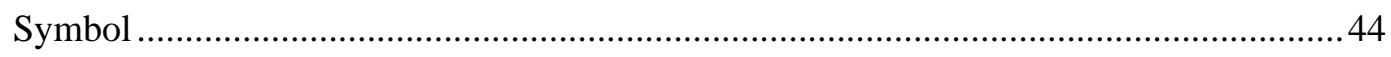

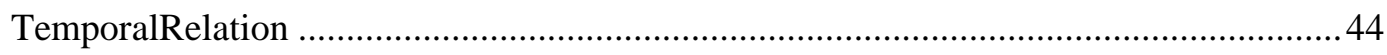

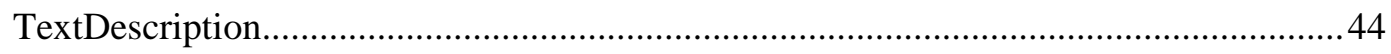

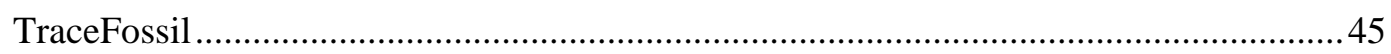

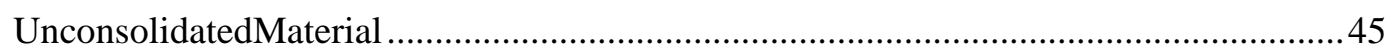

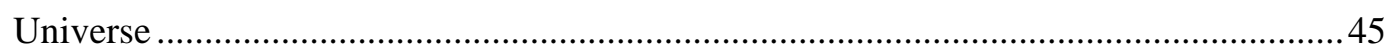

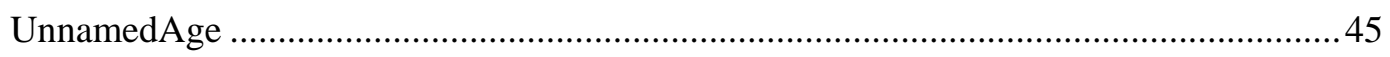

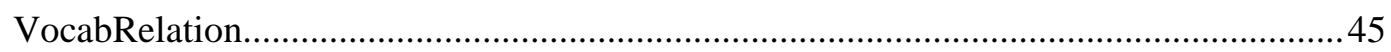

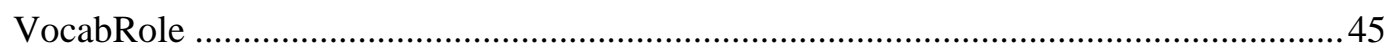

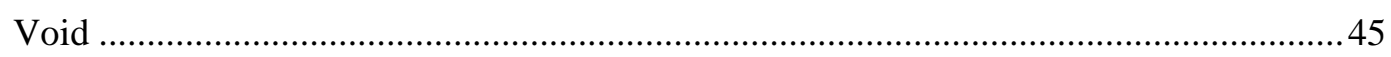

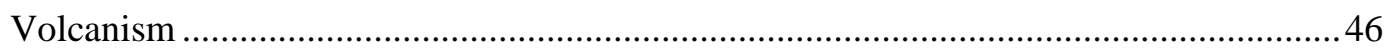

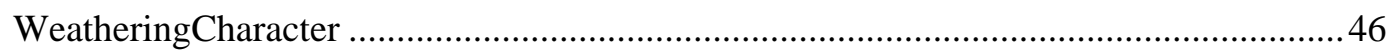




\section{Explanation of Abbreviations}

Derived from: Indicates parent class in class hierarchy

Attributes: List of attributes of a class in the model. Syntax is 'PropertyName: type', where the type may be another class in the model, a string, number, or enumerated list.

\section{AgeDate}

The age of a particular geologic event expressed in terms of years (absolute age), or by reference to a deposit representing some geologic instant.

AgeDate represents geologic instants, that is events or deposits that are treated as instantaneous relative to our ability to determine their duration or age. Isotopic ages are treated as representing the 'instant' of closure of some isotopic system in a rock, typically due to cooling. Examples of deposits representing geologic 'instants' include tuffs related to pyroclastic explosions and deposits related to bolide impacts.

\section{Derived from GeologicAge}

\section{AgeInterval}

The age of a particular geologic event or feature referred to the geologic time scale and expressed in terms of a range of years (absolute age) or of comparison with other geologic events or features (relative age). Intervals can be unnamed (i.e. 150-50 ma) or named (Cretaceous).

\section{Derived from GeologicAge}

\section{AllostratigraphicUnit}

A body of sedimentary rock that is distinguished by its bounding discontinuities. [NACSN, 1983] Must be mappable. Examples of boundaries are faults, disconformities, and paleosols.

\section{Derived from GeologicUnit}

\section{BaseMap}

A graphic used to provide geographic reference for a GeologicPortrayal MapDescription. A map description may have more that one base map. For example the eastern and western parts of a map may use different base maps. The base map for a CrossSection consists of the horizontal and vertical scale bars/bounding box, along with the topographic profile. Base maps have metadata properties (not modeled here) specifying the source of the image.

Derived from Graphic

\section{BeddingSurface}

A planar or nearly planar surface that visibly separates each successive layer of stratified rock (after Jackson, 1997). Construed narrowly to not generally include bed forms such as cross bedding.

Derived from PreLithificationStructure

\section{BiostratigraphicAge}

A NamedAge defined by fossil content in some rock body.

\section{Derived from NamedAge}




\section{BiostratigraphicUnit}

A biostratigraphic unit is a body of EarthMaterial defined or characterized by its fossil content.. [NACSN, 1983] Definition is independent of lithology and age, but biostratigraphic units commonly represent rock deposited during a particular time interval.

\section{Derived from GeologicUnit}

\section{Cast}

A replica or reproduction of the external details (size, shape, surface features) of a fossil shell, skeleton, or other organic structure, produced by the filling of a cavity originally formed by the decay or dissolution of some or all of the original hard parts of the organism. May form by in situ replacement; there may never be an actual cavity.

\section{Derived from Fossil, CompoundMaterial}

\section{ChemicalDescription}

A description of an EarthMaterial in terms of chemical constituents (oxides, elements...). Includes analyses and normative compositions. Details of the structure of these descriptions are beyond the scope of NADM-C1.

Derived from GeologicProperty

\section{ChronostratigraphicUnit}

A body of rock designated to serve as the material reference for all rocks formed during the same period of time [NACSN, 1983]. Each boundary is synchronous and therefore does not necessarily conform to lithostratigraphic or biostratigraphic units

\section{Derived from GeologicUnit}

\section{ClassSymbol}

A kind of LegendItem that associates a Symbol with a GeologicClass. Associates a Symbol (color, pattern, design, character, etc.) with a GeologicClass that specifies a collection of Occurrences displayed on a GeologicMap or CrossSection.

\section{Derived from LegendItem}

\section{Color}

An attribute to describe the color of an instance of a concept such as EarthMaterial or GeologicUnit. A controlled vocabulary for color might be very useful.

\section{Derived from GeologicProperty}

\section{CompoundFabric}

A Fabric composed of more than one GeologicStructure. Possible constituent structures include Foliation, Fracture, Layering, Lineation and other Fabrics. This constraint on possible constituents is not explicitly noted in the model schema. Example: Fabric including a foliation and a lineation.

\section{Derived from CompoundGeologicStructure}

\section{CompoundGeologicStructure}

A GeologicStructure composed of more than one GeologicStructure. Examples: Fold consisting of limbs and hinge; fault zone consisting of several faults. 


\section{CompoundMaterial}

An EarthMaterial composed of other EarthMaterial instances, possibly including other CompoundMaterial instances. Includes consolidated and unconsolidated materials as well as mixtures of consolidated and unconsolidated materials.

\section{Derived from EarthMaterial}

\section{ConsolidationDegree}

A property that specifies the degree to which an aggregation of EarthMaterial particles is a distinct solid material. Consolidation and induration are related concepts specified by this property. They define a continuum from noncemented collections of loose particles to very hard rock. Induration is the degree to which a consolidated material is made hard, operationally determined by how difficult it is to break a piece of the material. Consolidated materials may have varying degrees of induration.

\section{Derived from GeologicProperty}

\section{Contact}

A plane or irregular surface between two types or ages of EarthMaterial [adapted from page 137 of Jackson, 1997] Very general concept representing any kind of surface separating two EarthMaterials, including primary boundaries such as depositional contacts, all kinds of unconformities, intrusive contacts, and gradational contacts, as well as faults that separate geologic units.

\section{Derived from GeologicStructure}

\section{ControlledConcept}

A concept in the context of a GeologicVocabulary. A relationship class representing inclusion of an instance of a concept in a GeologicVocabulary. The GeologicConcept instance must have an associated definition, which may be text for human interpretation, or a formal database description following one of the description schema specified in NADM-C1. A ControlledConcept has exactly one preferred Name. VocabRelation specifies relationships between ControlledConcepts, which in the most common case are parent-child relationships establishing a concept hierarchy and synonymy establishing equivalent terms. Other Thesaurus type term relationships (BT, NT) could also be implemented.

\section{Derived from GeologicRepresentation}

\section{CorrelationChart}

A diagram used to demonstrate the correspondence in character and in stratigraphic position between geographically separated stratigraphic sections or rock bodies. Correlation charts are a kind of Graphic. The vertical axis in this Graphic typically has a connotation of time or thickness; the horizontal axis generally indicates some other attribute such as spatial separation.

\section{Derived from Graphic}

\section{CrossSection}

A diagram or drawing that shows features transected by a given plane. [Jackson, 1997] A CrossSection is essentially a geologic map with a vertical plane for a Horizon. It is not considered a Graphic because points in a cross section represent locations in the Earth. 


\section{Crystallization}

The process by which matter becomes crystalline, from a gaseous, fluid, or dispersed state. [Jackson, 1997]

\section{Derived from GeologicProcess}

\section{Deformation}

A process that results in a change in shape, location or orientation of a body of EarthMaterial [based on Means, 1976, p. 130]. Typical deformation processes include folding, faulting, and shearing. The concept includes prelithification and post-lithification processes.

\section{Derived from GeologicProcess}

\section{Deposition}

The constructive accumulation of any Earth material by a natural agent. Generally refers to sedimentary materials. Includes evaporation products and accumulations from the death of plants and animals.

\section{Derived from GeologicProcess}

\section{Diagenesis}

Any chemical, physical, or biological process that affects a sedimentary EarthMaterial after initial deposition, and during or after lithification, exclusive of weathering and metamorphism. [adapt. Jackson, 1997] Example processes include compaction, cementation, authigenesis, replacement, leaching, hydration, and bacterial action. Includes processes that are normal in the surficial or outer part of the earth's crust [Jackson, 1997]. Changes in a deeply buried sedimentary rock may be continuous from diagenesis into recrystallization to form a metamorphic rock.

Robertson [1999] defines the boundary between diagenesis and metamorphism in sedimentary rocks as follows:

“...the boundary between diagenesis and metamorphism is somewhat arbitrary and strongly dependent on the lithologies involved. For example changes take place in organic materials at lower temperatures than in rocks dominated by silicate minerals. In mudrocks, a white mica (illite) crystallinity value of $<0.42 \mathrm{D} .2 \mathrm{U}$ obtained by X-ray diffraction analysis, is used to define the onset of metamorphism (Kisch, 1991). In this scheme, the first appearance of glaucophane, lawsonite, paragonite, prehnite, pumpellyite or stilpnomelane is taken to indicate the lower limit of metamorphism (Frey and Kisch, 1987; Bucher and Frey, 1994; Frey and Robinson, 1998). Most workers agree that such mineral growth starts at $150 \pm 50^{\circ} \mathrm{C}$ in silicate rocks. Many lithologies may show no change in mineralogy under these conditions and hence the recognition of the onset of metamorphism will vary with bulk composition.”

Derived from GeologicProcess

\section{Discontinuity}

Fabric element that is a crack in the rock. Surface across which material cohesion/continuity in a rock is interrupted. May be open space, or filled with material introduced after formation of the discontinuity.

\section{Derived from GSDefiningElement}

\section{Displacement}

A measure of the change in position of material on one side of a fault relative to material on the other side of the fault. Includes both the direction of motion and the amount of movement. Subsumes the concepts separation and slip. 


\section{EarthMaterial}

A naturally occurring substance in the Earth. EarthMaterial represents substance, and is thus independent of quantity or location. Ideally, EarthMaterials are defined strictly based on physical and chemical properties, but because of standard geologic usage, genetic interpretations enter into the description as well. Melted rock (magma or lava) are not included in the context of this data model. Many concepts related to water or petroleum have not been modeled in this version.

\section{Derived from GeologicConcept}

\section{EMConstituent}

A relationship describing an EarthMaterial that is a part of some CompoundMaterial. Examples include: a mineral as a part of a rock, a leucocratic portion of a migmatite, the sand-sized portion of a laminated sand/shale sequence, groundmass in a porphyritic rock, etc. Although EMConstituent is a GeologicRelation in the model schema, it represents a physical thing (an EarthMaterial); it is a relationship because the essential aspect that differentiates constituent from material is the role of the constituent as a part of some whole (the CompoundMaterial)

\section{Derived from GeologicRelation}

\section{EMCRelation}

A relationship between two or more EMConstituents in a CompoundMaterial. An EMCRelation consists of a set of links between EMConstituents and the EMCRelation object. For example, EMCRelation specifies the type of relation (overgrowth) between constituents (albite and K-feldspar) in a CompoundMaterial (granite) in which albite forms overgrowths on K-feldspar phenocrysts.

\section{Derived from GeologicRelation}

\section{EMCRelationRole}

The role played by an EMConstituent in an EMCRelation. Specifies the standing of each EMConstituent involved in an EMCRelation to other EMConstituents participating in the relationship. Corresponds to 'AssociationEnd' names in a UML diagram. For example, in a relationship where albite forms overgrowths on K-feldspar phenocrysts, EMCRelation is 'overgrowth', the EMCRelationRole of albite in the relationship is 'encloses/overgrows', and the EMCRelationRole of K-feldspar is ‘enclosed/overgrown’.

\section{Derived from GeologicRole}

\section{EMCRole}

The role an EMConstituent plays in a CompoundMaterial aggregation. The same EarthMaterial may occur as different EMConstituents playing different roles within the same CompoundMaterial. For example, feldspar may be present as groundmass (an EMCRole) and as phenocrysts (an EMCRole) within a single igneous rock. Clast is a kind of EMCRole.

\section{Derived from GeologicRole}

\section{EMGenesis}

Represents the origin of an EarthMaterial as a sequence of GeologicEvents, each with an associated GeologicProcess in which the EarthMaterial is the final product. EMGenesis is derived from Genesis, which represents a geologic history as an ordered aggregation of GeologicEvents. 


\section{Erosion}

Process by which materials of the Earth's crust are loosened, dissolved, or worn away, and simultaneously moved from one place to another, by natural agencies. [Jackson, 1997] Erosion is a compound process, which includes both the breakdown of rock by weathering, solution, etc., and transportation of sediment away from the location of the original rock. Excludes: mass wasting.

\section{Derived from GeologicProcess}

\section{Extent}

A description of the spatial extent over which some phenomena may be observed. Used to describe the geographic area over which a geologic unit or structure may be found. May be described using point, line or polygon descriptions, depending on the nature of the phenomenon being described.

\section{Derived from GeologicProperty}

\section{Fabric}

Penetrative GeologicStructure defined by the relationship between constituent elements in an EarthMaterial. Fabric denotes a pattern, defined by one or more EMConstituents or GSDefiningElements, that is present throughout a rock body when considered at some scale. Fabric is defined based on the average configuration of many constituents. Penetrative denotes that these constituents are distributed throughout the rock volume at the scale of observation [Passchier and Trouw, 1998], and are repeated at distances that are small relative to the scale of the whole, such that they can be considered to pervade the whole uniformly and be present at every point (Turner and Weiss [1963] p. 21-24; Hobbs and others [1976], p. 73; Jackson [1997]; Passchier and Trouw [1998]). Fabric is distinguished from ParticleGeometry based on the criteria that ParticleGeometry is preserved if a CompoundMaterial is disaggregated, while Fabric is not defined if the material is disaggregated.

\section{Derived from GeologicStructure}

\section{Fault}

A discrete surface separating two rock masses across which one mass has slid past the other [based on Jackson, 1997].

SN: Restricted from definition of Jackson [1997] to include only a single surface. A fault zone, which includes several fault surfaces, is modeled as a CompoundGeologicStructure.

\section{Derived from GeologicStructure}

\section{Fold}

One or more systematically curved layers, surfaces, or lines in a rock body [adapted from Davis, 1984 and Hansen, 1971] "Folds are developed in rocks containing visible surfaces. Before folding, these surfaces may be plane or curved and adjacent surfaces may or may not be parallel” [quoted from Ramsay, 1967]. Fold denotes a structure formed by the deformation of a GeologicStructure to form a structure that may be described by the translation of an abstract line (the fold axis) parallel to itself along some curvilinear path (the fold profile). Folds have a hinge zone (zone of maximum curvature along the surface) and limbs (parts of the deformed surface not in the hinge zone). Folds are described by an axial surface, hinge line (ideally parallel to the fold axis abstraction), profile geometry, the solid angle between the limbs, and the relationships between adjacent folded surfaces if the folded structure is a Layering fabric (similar, parallel). Description of Fold is not modeled in detail. 


\section{Foliation}

A planar arrangement of textural or structural features in any type of rock. [Jackson, 1997] Includes any of a wide variety of penetrative planar geologic structures that may be present in a rock. Examples include schistosity, mylonitic foliation, penetrative bedding structure (lamination), and cleavage. Following the proposed definition of gneiss by the NADM Science Language Technical Team, penetrative planar foliation defined by layers $>5 \mathrm{~mm}$ thick is considered Layering. The committee discussed the possibility that layering should be considered a kind of foliation, but the majority opinion was that it is a different kind of structure.

\section{Derived from GeologicStructure}

\section{Fossil}

The remains, trace, or imprint of a life form that has been preserved in an EarthMaterial, and demonstrates evidence of having been changed from its original biogenic form. Fossil is distinguished from biologic remains or biogenic sedimentary structure based on evidence of having been converted incipiently or substantially into a modified version of the original biogenic form or structure. Although the passage of time is implicit in the definition of fossil, no constraint is placed on the amount of time necessary to become a fossil. Includes ichnofossils, casts and molds, as well as fossilized remains. Subtypes of fossil are also considered kinds of CompoundMaterial to represent their material aspect or kinds of GeologicStructure to represent their morphologic aspect.

\section{Derived from GeologicConcept}

\section{Fracture}

A crack in a body of EarthMaterial across which there is insignificant lateral displacement. Fractures may be open or filled with material introduced after cracking. The essential aspect of a fracture is its origin by brittle failure in an EarthMaterial. The model considers joint a kind of fracture. A fracture with significant displacement (determined based on the scale of observation/interest) would be considered a fault in NADM-C1.

\section{Derived from GeologicStructure}

\section{Genesis}

Represents geologic history as an ordered aggregation of GeologicEvent objects, each of which may have an associated GeologicAge, GeologicEnvironment, and one or more GeologicProcess objects. Genesis typically pertains to some geologic phenomenon (GeologicStructure, EarthMaterial, GeologicUnit, Fossil, etc.)

Derived from GeologicProperty

\section{GeologicAge}

The age of a particular geologic event or feature expressed in terms of years before present (absolute age), referred to the geologic time scale, or by comparison with other geologic events or features (relative age). A GeologicAge can represent an instant in time, an interval of time, or any combination of multiple instants or intervals. Combinations of instants and intervals are beyond the scope of NADM-C1. Specifications of age in years before present are based on determination of time durations based on interpretation of isotopic analyses of EarthMaterial (some other methods are used for geologically young materials). Ages referred to geologic time scales are essentially based on correlation of a geologic unit with a standard chronostratigraphic unit that serves as a reference (see NACSN, 1983). Relative ages are based on relationships between geologic units such as superposition, intruded by, cross-cuts, or 'contains inclusions of'. 


\section{GeologicClass}

GeologicClass represents a collection of objects associated with a specific GeologicConcept and shown on a map using a particular symbol. GeologicClass is thus a collection of Occurrences that correspond to a specific GeologicConcept and have a unique symbolization in a MapDescription. GeologicClass is distinct from GeologicConcept because some GeologicConcepts are symbolized using more than one symbol. This allows symbolization based on criteria in addition to the GeologicConcept. Examples: Geologic contacts maybe be symbolized differently based on location uncertainty; different bedding symbols are used for horizontal, inclined, vertical and overturned, but all are representative of the GeologicConcept 'BeddingSurface'.

\section{Derived from GeologicPortrayalComponent}

\section{GeologicConcept}

A subset of the Universe of all concepts, which includes only those concepts related to: "The study of the planet Earth--the materials of which it is made, the processes that act on these materials, the products formed, and the history of the planet and its life forms since its origin." [Jackson, 1997; p. 265] This conceptual model is focused primarily on geologic concepts that can be represented on geologic maps and diagrams. The term concept represents the notion of any mental phenomena that human beings use in their internal representation of the world. Webster's dictionary [1996] uses the terms 'idea' and 'object of thought' to convey the meaning of 'concept.' GeologicConcepts identify the kinds of observable or inferred phenomena that earth scientists recognize.

\section{Derived from Universe}

\section{GeologicEnvironment}

The physical setting within which a GeologicEvent takes place. GeologicEnvironment is construed broadly to include physical settings on the Earth surface specified by climate, tectonics, physiography or geography, and settings in the Earth's interior specified by pressure, temperature, chemical environment, or tectonics. Specification of setting may be a simple text description or a link to a complex description.

\section{Derived from GeologicProperty}

\section{GeologicEvent}

An identifiable event during which one or more geologic processes act to modify geologic entities. A GeologicEvent may have a specified GeologicAge and GeologicEnvironment.. An example might be a cratonic uplift event during which erosion, sedimentation, and volcanism all take place.

\section{Derived from GeologicConcept}

\section{GeologicMap}

A map on which is recorded geologic information, such as the distribution, nature, and age relationships of geologic units, and the occurrence of structural features, mineral deposits, and fossil localities. [Jackson, 1997] In NADMC1, GeologicMap is restricted to the graphical element that displays geologic information according to a mathematical mapping with some Earth coordinate, such that locations on the map correspond to locations on (or in) the Earth. The map is incomplete as a means of communicating information without an associated Legend and LegendGraphic.

\section{Derived from GeologicPortrayal}

\section{GeologicPortrayal}

A visualization designed to convey geologic information. GeologicPortrayal includes GeologicMap and CrossSection, as well as Graphic. 


\section{GeologicPortrayalComponent}

Models concepts that are parts of the description of a GeologicPortrayal. An abstract class that is the parent of: MapLayout, MapDescription, Legend, LegendItem, GeologicClass, Occurrence and SpatialObject.

Derived from GeologicRepresentation

\section{GeologicProcess}

A GeologicProcess is a function, possibly complex, that acts on one geologic entity to produce another geologic entity at a later time. GeologicProcess is time independent; some GeologicProcesses are observable in the present at work in the world or in the laboratory, others can only be inferred from observing the results of the process. Processes take one or more of EarthMaterial, GeologicUnit, or GeologicStructure as input and have one or more of EarthMaterial, GeologicUnit or GeologicStructure as output.

Derived from GeologicConcept

\section{GeologicProperty}

An inherent feature used to characterize a GeologicConcept.

\section{Derived from GeologicConcept}

\section{GeologicRelation}

Represents any of a wide variety of relationships that can exist between two or more GeologicConcepts. For example, the GeologicRelation 'intrudes' is a relationship between an intrusive igneous rock and some host rock. Includes spatial, temporal, sequence, correlation, and parent/child relations. Many of the relationships in NADM-C1 (particularly attribute links and parent-child links) are not explicitly modeled as kinds of GeologicRelation.

Derived from GeologicConcept

\section{GeologicRepresentation}

Abstract superclass for concepts related to representation of geologic knowledge. GeologicRepresentation concepts represent things that are human artifacts, invented for the purpose of representing conceptions of the Earth on paper, in text, or in computer information systems.

Derived from Universe

\section{GeologicRole}

Identifies the role of a GeologicConcept in a GeologicRelation. Specifies the standing of each GeologicConcept involved in a GeologicRelation to other GeologicConcepts participating in the relationship. Corresponds to 'AssociationEnd' names in a UML diagram. GeologicRole represents a GeologicConcept in the context of a particular relationship. For example, in a relationship where an igneous unit intrudes a sedimentary unit, GeologicRelation is 'intrudes', the intruded sedimentary unit has the GeologicRole 'host', and the igneous unit has the GeologicRole ‘intrusion’.

Derived from GeologicProperty

\section{GeologicStructure}

A configuration of matter in the Earth based on describable inhomogeneity, pattern, or fracture in an EarthMaterial The identity of a GeologicStructure is independent of the material that is the substrate for the structure. There are 
almost always strong dependencies between the nature of the EarthMaterial substrate and the kinds of GeologicStructure that may be present. A disaggregated heap of particles does not have structure, and can only be described in terms of the mineralogy and geometrical character of the constituent particles. GeologicStructures are more likely to be found in, and are more persistent in, consolidated materials than in unconsolidated materials. Properties like 'clast-supported', 'matrix-supported', and 'graded bed' that do not involve orientation are considered kinds of GeologicStructure because they depend on the configuration of parts of a rock body. Includes: sedimentary structures.

\section{Derived from GeologicConcept}

\section{GeologicTimeScale}

A hierarchical GeologicVocabulary that partitions the domain of geologic time at several different levels (eon, era, period, epoch, etc.).

\section{Derived from GeologicVocabulary}

\section{GeologicUnit}

A body of EarthMaterial distinguished from adjoining EarthMaterial on the basis of some stated property or properties [adapted from NACSN, 1983, p.22; http://www.agiweb.org/nacsn/code2.html]. Corresponds to 'stratigraphic unit' in the North American Stratigraphic Code. A volumetric body of EarthMaterial distinguished by content (lithologic or fossil), inherent attributes, physical limits, or geologic age. Commonly used properties include composition, texture, included fossils, magnetic signature, radioactivity, seismic velocity, and age. Sufficient care is required in defining the boundaries of a unit to enable others to distinguish the material body from those adjoining it [NACSN, 1983].

A GeologicUnit is a part of the solid Earth that is identified by its geologic characteristics, has definable, locatable boundaries, and is persistent in time. Excludes non-material, temporal units. GeologicUnits are related using the general GeologicRelation structure.

Derived from GeologicConcept

\section{GeologicUnitPart}

A GeologicRelation that links constituent parts of a GeologicUnit with the whole GeologicUnit in a description of a GeologicUnit as an aggregation of parts. Parts of GeologicUnits may be other GeologicUnits or CompoundMaterials.

UML Note: The associations between GeologicUnitPart --CompoundMaterial and GeologicUnitPart -GeologicUnit are IsA or subtype relationships, but our modeling tool (Rational Rose 98) will not allow such a construction. The disjoint association with CompoundMaterial and GeologicUnit is used because some GeologicUnits have only one CompoundMaterial as a constituent. In order to use the recursive aggregation constuction as in EarthMaterial (Plate 2), a single-lithology GeologicUnit would have to have a single GeologicUnitPart composed of a single CompoundMaterial.

Derived from GeologicRelation

\section{GeologicVocabulary}

A collection of terms and their associated definitions (GeologicConcepts), usually organized in some logical fashion such as in a hierarchy (geologic time, rocks, unconsolidated deposits...). An instance of a GeologicConcept may occur only once in a particular GeologicVocabulary, and in the context of the vocabulary (represented by 'ControlledConcept' object), it has exactly one preferred Name (preference link in schema for GeologicVocabulary, Plate 11). 


\section{GeometricDescription}

Description of the geometry of a geologic structure. GeometricDescription includes: Orientation, Thickness, Planar/Linear, Fold wavelength and amplitude, etc. In detail, this description will have different attributes and constraints for different types of geologic structures. Details of coding these parameters are beyond the scope of NADM-C1.

\section{Derived from GeologicProperty}

\section{GeomorphologicUnit}

A GeologicUnit defined by surface landforms and the associated Earth processes that formed them. Common usage in surficial deposit mapping. Although these units are largely defined and identified based on surface morphology, they always have some material volume associated with them. They are similar to allostratigraphic units, but a lower bounding surface is not defined [NACSN, 1983, p.66].

\section{Derived from GeologicUnit}

\section{Glass}

Solid inorganic material intermediate between the close-packed, highly ordered array of a crystal and the poorly packed, highly disordered array of a gas. Generally the product of the rapid cooling of a magma. Includes: volcanic glasses and clinkers.

Excludes: chert and other cryptocrystalline materials.

Derived from InorganicFluid

\section{Graphic}

Represents figures designed to convey information visually, but in which the arrangement of graphic elements is not a mapping (in the technical sense) to a location on/in the Earth, but may follow some other logic. Correlation charts, explanations of map units, ternary diagrams to show composition, and text blocks are examples of Graphic. Includes raster images and vector representations. May be displayed digitally (on screen) or by printing or plotting.

Derived from GeologicPortrayalComponent

\section{GSDefiningElement}

Kinds of describable inhomogeneity in a rock body that may define a GeologicStructure. Examples include Discontinuity, ShapedSurface, OrientedParticle, MaterialBoundary, and Layer. The details of structure description as a composite entity with these parts have not been modeled.

Derived from GeologicProperty

\section{GSGenesis}

Represents the origin of a GeologicStructure as a sequence of GeologicEvents, each with an associated GeologicProcess acting on an input GeologicStructure, GeologicUnit, or EarthMaterial to produce an output GeologicStructure.

\section{Derived from Genesis}




\section{GUGenesis}

Represents the origin of a GeologicUnit as a sequence of GeologicEvents, each with an associated GeologicProcess acting on an input GeologicUnit or EarthMatertial to produce an output GeologicUnit. The GeologicUnit associated with the GUGenesis is the product of the final GeologicProcess in the sequence. In many cases, the input GeologicUnit or EarthMaterial for the GeologicProcess associated with a GUGenesis object is hypothetical, because it has become the GeologicUnit that is the product of the GeologicProcess.

Derived from Genesis

\section{GUPRelation}

Kinds of relationships between two or more GeologicUnitParts in a GeologicUnit. For example, the GUPRelation 'overlies' is a relationship between parts of a GeologicUnit in which one is above the other. Examples: underlies, overlies, intrudes, grades laterally, etc.

\section{Derived from GeologicRelation}

\section{GUPRelationRole}

Identifies the role of a GeologicUnitPart in a GUPRelation. Specifies the standing of each GeologicUnitPart involved in a GUPRelation to other GeologicUnitPart objects participating in the relationship. Corresponds to 'AssociationEnd' names in a UML diagram. GUPRelationRole represents a GeologicUnitPart in the context of a particular GUPRelation. For example, in a relationship where one part of a geologic unit overlies another, GUPRelation is 'overlies', the overlying GeologicUnitPart has the GUPRelationRole 'UpperPart', and the overlain GeologicUnitPart has the GUPRelationRole 'LowerPart'.

Derived from GeologicRole

\section{GURole}

The role a GeologicUnitPart plays in the GeologicUnit. The same EarthMaterial (sandstone) may occur as different GeologicUnitParts playing different roles within the same GeologicUnit. For example, sandstone may be a member of the geologic unit as well as being interbedded with a shale member. Examples include: member, vein, interbed, etc.

\section{Derived from GeologicRole}

\section{Horizon}

For a 2-D (planar) GeologicMap or CrossSection, Horizon represents a surface (possibly abstract) within or on the Earth that contains the geologic traces depicted in the visualization. Geologic surfaces in the Earth (faults, contacts, fold hinge surfaces, dikes...) intersect the Horizon to define a trace; this trace is located, and projected at some scale to determine the lines (SpatialObjects) depicted on a map. This concept can be extended to 3-D by considering the Horizon to be the volume in the Earth that contains the features depicted.

Derived from GeologicPortrayalComponent

\section{HydrothermalAlteration}

The alteration of rocks or minerals by the reaction of hydrothermal fluid with pre-existing solid phases. [Jackson, 1997]

\section{Derived from GeologicProcess}




\section{InorganicFluid}

An inorganic EarthMaterial (solid, liquid, or gas) that tends to flow or conform to the shape of its container. Includes glass. By convention liquid mercury is considered a mineral

\section{Derived from EarthMaterial}

\section{Intrusion}

The process of injection of unlithified Earth material into pre-existing rock or sediment. [DMDT] Includes magma in the igneous sense, as well as sedimentary dikes and diapirs.

Derived from GeologicProcess

\section{IntrusiveRelation}

A GeologicRelation that involves the spatial or temporal relationships of cross cutting rock bodies caused by the process of injection of unlithified Earth material into pre-existing rock or sediment. [DMDT]

\section{Derived from GeologicRelation}

\section{Joint}

A planar fracture in a rock, without shear displacement, that is part of a systematically oriented set of fractures. Joint is a subclass of fracture, distinguished by its occurrence as part of a collection of similarly oriented fractures.

\section{Derived from Fracture}

\section{Layer}

A geologic structure element (GSDefiningElement) that is a sheet-like part of the rock characterized by mineralogy or texture.

\section{Derived from GSDefiningElement}

\section{Layering}

Planar foliation defined by a tabular succession of layers $>5 \mathrm{~mm}$ thick. This definition is based on the proposed definition of gneiss by the NADM Science Language Technical Team, so that the GeologicStructure characteristic of gneiss is layering. The committee discussed the possibility that layering should be considered a kind of foliation, but the majority opinion was that it is a different kind of structure.

\section{Derived from GeologicStructure}

\section{Legend}

An ordered collection of LegendItems. A MapDescription includes one or more Legend objects that specify the symbols (including patterns and colors) displayed on a GeologicMap or CrossSection, and their meaning. The ordering of LegendItems specifies an arrangement of the Symbol/Explanation pairs in the LegendGraphic.

\section{Derived from GeologicPortrayalComponent}

\section{LegendGraphic}

LegendGraphic is a Graphic that displays the Legend or Legends associated with a MapDescription. A GeologicMap or CrossSection visualization must be accompanied by a LegendGraphic to convey the meaning of the symbols on the map. The LegendGraphic image visually associates symbols with concepts for human map-readers. 


\section{LegendItem}

A relationship class that represents the association of a GeologicClass with a Symbol or a SpatialObject with a Symbol. The meaning of the symbol is implicitly linked through the association of the GeologicClass with a GeologicConcept, or association of a SpatialObject with an Occurrence and the Occurrence with a GeologicConcept. Each LegendItem represents a single entry in the GeologicMap or CrossSection Legend. Each LegendItem describes either a single entity (occurrence) on the map or section or a single class of entities occurring on the map or section. Includes: map units, structural symbols, localities (i.e. mines, fossils, etc.).

\section{Derived from GeologicPortrayalComponent}

\section{Lineation}

GeologicStructure defined by aligned elongate elements. Lineation connotes a pervasive linear structure in a body of EarthMaterial or on a fracture surface. Includes: flow lines, scratches, striae, slickenlines, linear arrangements of elongate components in sediments, fold hinges (when abundant and closely spaced), elongate minerals, crinkles, and lines of intersection between penetrative planar structures.

\section{Derived from GeologicStructure}

\section{LithodemicUnit}

A body of predominantly intrusive, highly deformed, and/or highly metamorphosed rock distinguished on the basis of lithic characteristics. [NACSN, 1983] Generally lacks primary stratification. Definition is based on lithology, not on genesis. Lithodemic units generally do not conform to the law of superposition. Contacts of lithodemic units with other rock units may be sedimentary, extrusive, intrusive, tectonic, or metamorphic.

\section{Derived from GeologicUnit}

\section{LithologyClassification}

A hierarchical GeologicVocabulary that subdivides the domain of CompoundMaterials. Typically includes a hierarchical arrangement of named classes at several different levels of generality. Examples of different levels include: igneous rock, granitic rock, granite. The classification must include definitions of the included classes specifying how to determine the class to which a given CompoundMaterial belongs.

\section{Derived from GeologicVocabulary}

\section{LithostratigraphicUnit}

A body of sedimentary, extrusive igneous, metasedimentary, or metavolcanic strata distinguished on the basis of lithic characteristics and stratigraphic position. [NACSN, 1983, article 22] Generally conforms to the law of superposition. The definition of a Lithostratigraphic unit is independent from geologic history and time (although there may be a close correlation between the unit and a geologic history or age). Lithostratigraphic units are recognized and defined by observable rock characteristics; boundaries may be placed at clearly distinguished contacts or drawn arbitrarily within a zone of gradation. Lithification or cementation is not a necessary property; clay, gravel, till, and other unconsolidated deposits may constitute valid lithostratigraphic units.

\section{Derived from GeologicUnit}

\section{LithotectonicUnit}

An assemblage of rocks that is unified on the basis of structural or deformational features, mutual relations, origin, or historical evolution. [Jackson, 1997] Can be igneous, sedimentary, or metamorphic rock. Classification not included in North American Stratigraphic code, but commonly used for continental-scale maps. 


\section{MagnetostratigraphicAge}

A time interval defined with reference to a polarity-chronostratigraphic unit [NACSN, 1983, article 88] Corresponds to polarity-chronologic unit of NACSN [1983]. A different name is used here to avoid confusion between 'unit' as a time interval or rock body. No special kind of magnetic time is implied; the designations used are meant to convey the parts of geologic time during which the Earth's magnetic field had a characteristic polarity or sequence of polarities. These units correspond to the time spans represented by polarity chronozones, e.g., Gauss Normal Polarity Chronozone. They are not material units. Care should be taken to define polarity-chronologic units in terms of polarity-chronostratigraphic units, and not vice versa. [NACSN, 1983].

\section{Derived from NamedAge}

\section{MagnetostratigraphicUnit}

A body of rock unified by specified remanent-magnetic properties and distinct from underlying and overlying magnetostratigraphic units having different magnetic properties. [NACSN, 1983, article 43] Four basic paleomagnetic phenomena can be determined or inferred from remanent magnetism: polarity, dipole-field-pole position (including apparent polar wander), the non-dipole component (secular variation), and field intensity [NACSN, 1983]. Definition of a MagnetostratigraphicUnit is independent of genesis.

\section{Derived from GeologicUnit}

\section{MapDescription}

This class represents a specification of the spatial data, geologic classification, and symbolization that together record the construction of a particular GeologicMap or CrossSection. A MapDescription records information necessary to reconstruct a geologic map or cross section and a legend graphic, but does not specify accompanying Graphic objects (CorrelationChart, StratigraphicColumn) or explanatory text.

\section{Derived from GeologicPortrayalComponent}

\section{MapLayout}

Represents a collection of graphical objects that constitute a geologic map as well as the arrangement of those objects in a map visualization. Must include at least one MapDescription object-either a GeologicMap or CrossSection, and may include additional Graphic objects including text, photographs, figures, LegendGraphic, CorrelationChart, etc.

\section{Derived from GeologicPortrayalComponent}

\section{MaterialBoundary}

Geologic structure defining element (GSDefiningElement) defined by a discernible boundary between EarthMaterials with different properties (grain size, composition, sorting, fabric...)

\section{Derived from GSDefiningElement}

\section{Metadata}

Additional information about the geologic database or portions of the database (i.e. a map) that is not included in the model. Includes background information such as, author, organization, creation date, methodology, etc. 


\section{MetamorphicGrade}

The intensity or rank of metamorphism. Indicates in a general way the P-T environment in which the metamorphism took place. May be applied to an EarthMaterial or GeologicUnit. Determination of metamorphic grade is based on mineral assemblages present in a rock that are interpreted to have crystallized in equilibrium during a particular metamorphic event.

\section{Derived from GeologicProperty}

\section{Metamorphism}

GeologicProcess by which solid rocks adjust to physical and chemical conditions that differ from the conditions under which the rocks in question originated. [based on Jackson, 1997] Includes mineralogical, chemical, and structural changes. Applied to conditions that have generally been imposed deeper in the Earth than the surface zones of weathering and cementation. [based on Jackson, 1997]

\section{Derived from GeologicProcess}

\section{Metasomatism}

The process of practically simultaneous dissolution and deposition by which a new mineral of partly or wholly different chemical composition may grow in the body of an old mineral or mineral aggregate. [Jackson, 1997] Generally occurs with little volume change or textural disturbance.

\section{Derived from GeologicProcess}

\section{Mineral}

A naturally occurring inorganic element or compound having a periodically repeating arrangement of atoms and a characteristic chemical composition or range of compositions, resulting in distinctive physical properties. Includes mercury as a general exception to the requirement of crystallinity. Also includes cryptocrystalline materials such as chalcedony and amorphous silica.

\section{Derived from EarthMaterial}

\section{Mold}

Impressions or cavities in rock or sediment matrix having the gross morphology of a fossil organism, formed when the buried organism's original material was removed by dissolution.

\section{Derived from Fossil, GeologicStructure}

\section{Morphology}

The geometry or form of a GeologicUnit. Examples include: dike, cone, fan, sheet, etc. Morphology is independent of the substance (EarthMaterial) that composes the GeologicUnit.

\section{Derived from GeologicProperty}

\section{Name}

Name is an attribute of all GeologicConcepts. Therefore, any instance of any concept can be labeled with one or more short names. Intended as a location to store single word or short phrase descriptors of individual instances of any concept. 


\section{NamedAge}

An interval of time defined by a material referent [NACSN, 1983]. This concept corresponds to geochronologic unit of North American Stratigraphic Code [NACSN, 1983]. A different name is used here to avoid confusion as to whether the term refers to a rock body or interval of time. It has a specific time span, which can only be measured or calibrated approximately. A NamedAge corresponds to the interval of time represented by an established chronostratigraphic unit (Articles 65 and 66), and the beginning and ending of the interval correspond to the base and top of the referent [NACSN, 1983, article 80]. More generally this is taken to mean the beginning and ending of the interval correspond to events that started and ended deposition, crystallization or metamorphism of a sedimentary, igneous, or metamorphic rock, respectively. Examples: Jurassic, Precambrian, Cretaceous Long Normal Polarity Chron, Polarity Chron C33n

Derived from AgeInterval

\section{Occurrence}

An Occurrence is a single instance of the collection of data items represented by a GeologicClass.

Each Occurrence differs from all other Occurrences either in some portion of its definition (GeologicConcept), or in its geometry (size, shape, location). For example, two different outcrops of the same map unit (same GeologicConcept) are different Occurrences because they differ in geometry (location). Similarly, the representations of the outcrop trace of the same fault (same GeologicConcept) at different scales are different Occurrences because the geometry (size) is different. Features which share the same geometry, but different definitions, such as a fault and a contact that are represented by the same line on a map, are also unique Occurrences. An Occurrence is always associated with a GeologicConcept, either directly or indirectly through a GeologicClass. In order to be depicted on a GeologicPortrayal (GeologicMap or CrossSection), an occurrence must be associated with a SpatialObject. Typically, a GeologicClass is associated with many Occurrences.

\section{Derived from GeologicPortrayalComponent}

\section{OccurSymbol}

A kind of LegendItem that associates a Symbol with a single SpatialObject. This relationship is used to assign symbols (color, pattern, design, character, etc.) to individual occurrences on a map or section, independent of their assignment to a GeologicClass. Symbol assignment by an OccurSymbol instance takes precedence over symbol assignment by link from Occurrence to GeologicClass. This link is necessary 1) to symbolize Occurrences that have instance attributes that enter into symbolization rules, or 2) when for cartographic reasons an Occurrence assigned to some GeologicClass must be symbolized with a different symbol. An example of case 1 is bedding symbols that must be rotated according to the azimuth of the strike. Examples of case 2 include a mine shaft location that is symbolized uniquely because that location is described in detail in the legend, or polygons of a map unit that are too small to display and are cartographically dissolved by giving them the same symbol as adjacent polygons.

\section{Derived from LegendItem}

\section{OrganicMaterial}

An EarthMaterial that belongs to the class of chemical compounds having a reduced carbon basis (as distinct from carbonates), and derived from living organisms. Includes high-carbon EarthMaterials such as bitumen, peat, and coal.

\section{Derived from EarthMaterial}




\section{OrientedParticle}

Element of geologic structure defined by the orientation of the long axis of a prolate particle or the plane defined by an oblate particle.

\section{Derived from GSDefiningElement}

\section{OutcropCharacter}

Describes the nature of outcrops formed by a geologic unit. Examples: bouldery, cliff-forming, ledge-forming, slope-forming, poorly exposed

\section{Derived from GeologicProperty}

\section{ParticleGeometry}

The bulk characterization of particles in an aggregate, independent of their relationship to each other or orientation. ParticleGeometry describes the particle size, particle size distribution, and particle shape, and particle shape distribution. ParticleGeometry may have properties such as: particle sorting, size, size variation, shape, and possibly others. It is applied to megascopic and microscopic features best seen on the smooth surface of a rock or within an unconsolidated material. ParticleGeometry is distinguished from fabric in that the ParticleGeometry description of a CompoundMaterial remains constant if the material is disaggregated into its constituent particles, whereas fabric is lost if the material is disaggregated.

Note that because Void (pore space) is modeled as a potential constituent, pore geometry is described using the ParticleGeometry construct. Some practitioners call this concept Texture.

\section{Derived from GeologicProperty}

\section{ParticleShape}

The bulk characterization of the shape of the particles in an aggregate. This property may describe the shape of particles in a CompoundMaterial as a whole, or the shape of the particles of a particular EMConstituent. May use terminology (roundness, sphericity), or include quantitative shape parameters. These descriptions are not modeled in detail

\section{Derived from GeologicProperty}

\section{ParticleSize}

The bulk characterization of the size of particles in an aggregate. This property may describe the size distribution of particles in a CompoundMaterial as a whole, or the size distribution of the particles of a particular EMConstituent. The size description includes information about grain size and grain size distribution. May take the form of terminology (fine-grained, poorly sorted), scalar parameters (mean grain size, standard deviation, kurtosis...), or a grain-size analysis as a histogram of size vs. frequency.

\section{Derived from GeologicProperty}

\section{Pedoderm}

A mappable mantle of soil, entirely or partially truncated, at the Earth's surface or partially or wholly buried, which has physical characteristics and stratigraphic relationships that permit its consistent recognition and mapping. [Jackson, 1997] May be modified or even made by humans. Apparently has replaced Pedologic Unit of American Code of Stratigraphic Nomenclature [1970], which does not appear in NACSN [1983].

Pedoderm is a proposed INQUA-ISSS (International Union for Quaternary Research and International Society of Soil Science. Parsons, 1981) soil-stratigraphic unit. A pedoderm is a surficial soil; may be a buried, relict, or 
exhumed soil; a pedoderm is the entire soil (from NACSN, 1983, p. 28). See also Brewer and others [1970] and Walker and others [1984].

\section{Derived from GeologicUnit}

\section{PedostratigraphicUnit}

A buried, traceable, three-dimensional body of EarthMaterial that consists of one or more differentiated pedologic horizons and is overlain by one or more formally-defined lithostratigraphic or allostratigraphic units. [adapted from NACSN. 1983, Article 55]. Developed in one or more lithostratigraphic, allostratigraphic, or lithodemic units. Distinct from pedoderm in that they are not entirely composed of pedogenic materials.

"A pedostratigraphic unit differs from other stratigraphic units in that (1) it is a product of surface alteration of one or more older material units by specific processes (pedogenesis), (2) its lithology and other properties differ markedly from those of the parent material(s), and (3) a single pedostratigraphic unit may be formed in situ in parent material units of diverse compositions and ages" [NACSN, 1983].

\section{Derived from GeologicUnit}

\section{Pervasiveness}

The degree to which a GeologicStructure occurs as a discrete entity or is distributed (penetrative) throughout an EarthMaterial. End members are discrete and penetrative. Discrete structures have a particular location, and some unity criteria that allows identification of the entire structure as a unit. Particular faults are discrete structures. Penetrative denotes that these constituents are distributed throughout the rock volume at the scale of observation [Passchier and Trouw, 1998], and are repeated at distances that are small relative to the scale of the whole, such that they can be considered to pervade the whole uniformly and be present at every point (Turner and Weiss [1963] p. 21-24; Hobbs and others [1976], p. 73; Jackson [1997]; Passchier and Trouw [1998]). Structures may be penetrative at some scales and discrete at others.

Derived from GeologicProperty

\section{PhysicalProperty}

A characteristic of an EarthMaterial or GeologicUnit that is intrinsic to its substance and can be characterized by some physical measurement. Includes properties like density, sound velocity, and magnetic susceptibility. Details of coding these parameters are beyond the scope of NADM-C1.

\section{Derived from GeologicProperty}

\section{PolarityChronostratigraphicUnit}

A body of rock that contains the primary magnetic-polarity record imposed when the rock was deposited or crystallized during a specific interval of geologic time. [NACSN, 1983] "Polarity-chronostratigraphic units depend fundamentally for definition on actual sections or sequences, or measurements on individual rock units, and without these standards they are meaningless. They are based on material units, the polarity zones of magnetopolarity classification. Each polarity-chronostratigraphic unit is the record of the time during which the rock formed and the Earth's magnetic field had a designated polarity.” [NACSN, 1983]

Derived from GeologicUnit

\section{PreLithificationStructure}

A Structure created before lithification of the rock that contains the structure. [paraphrased from page 510 of Jackson, 1997] Includes soft-sediment deformation and other secondary sedimentary structures occurring prior to lithification; structures that form in igneous rocks before lithification.

UF: bed form, primary sedimentary structures, primary igneous structures 


\section{PreservedRemain}

The original materials comprising an organism's body, shell, or other hard parts. Includes recrystallized but not replaced material. Also includes non-fossilized bone and shell remains. This material would become fossil material when it is deemed to be 'preserved ... since some past geologic or prehistoric time' [Jackson, 1997].

\section{Derived from Fossil, CompoundMaterial}

\section{Projection}

The mathematical mapping between locations on or in the Earth and a GeologicPortrayal.

\section{Derived from GeologicProperty}

\section{Proportion}

The fraction of the whole that is formed by a part in a part/whole relationship. Used for the EMConstituent portion in a CompoundMaterial and for the GeologicUnitPart portion of a GeologicUnit. Typically represented by a value in the domain (0..1). In general the value would be a general quantity object that may be a single value with uncertainty, a range of values expressed by a max and min, a qualitative value (rare, common...), or a relative value (most abundant, least abundant...).

\section{Derived from GeologicProperty}

\section{QualitativePSD}

A qualitative, textual description of grain size and size distribution of particles forming a CompoundMaterial or of particles of a particular EMConstituent within a CompoundMaterial (e.g. fine-grained, poorly sorted, etc.).

\section{Derived from GeologicProperty}

\section{QualitativePShD}

A qualitative, textual description of grain shape of particles forming a CompoundMaterial or of particles of a particular EMConstituent within a CompoundMaterial (e.g. well-rounded, angular, etc.).

Derived from GeologicProperty

\section{QuantitativePSD}

A quantitative description of the particle size and/or size distribution of particles forming a CompoundMaterial or of particles of a particular EMConstituent within a CompoundMaterial. Includes scalar parameters (mean grain size, standard deviation, kurtosis...), or a grain-size analysis as a histogram of size vs. frequency. Details of coding these parameters are beyond the scope of NADM-C1.

\section{Derived from GeologicProperty}

\section{QuantitativePShD}

A quantitative description of the particle shape of particles forming a CompoundMaterial or of particles of a particular EMConstituent within a CompoundMaterial. Includes scalar parameters (degree of roundness, etc.), or a more detailed grain-shape analysis. Details of coding these parameters are beyond the scope of NADM-C1. 


\section{RadiometricAge}

An AgeDate determined based on the decay of radioactive isotopes.

\section{Derived from AgeDate}

\section{Rank}

A partial ordering property of GeologicUnit. Orders geologic units based on extent of unit or inclusion of other geologic units. Examples include: group, formation, bed, etc.

\section{Derived from GeologicProperty}

\section{Rock}

A consolidated aggregate of one or more EarthMaterials, or a body of undifferentiated mineral matter, or of solid organic material [adapted from Jackson, 1997]. Includes mineral aggregates such as granite, shale, marble; mineral matter, such as obsidian (Glass); and organic material, such a coal. Excludes unconsolidated materials.

\section{Derived from CompoundMaterial}

\section{Sample}

Represents a material sample or physical specimen.

\section{Derived from Occurrence}

\section{Scale}

The mathematical relationship between length in the portrayal space and length in the earth.

\section{Derived from GeologicProperty}

\section{Sedimentation}

The process of depositing sediment by mechanical means from a state of suspension in air or water. [Jackson, 1997] A limited definition to exclude other processes related to the erosion, deposition, and lithification of sediment.

\section{Derived from Deposition}

\section{ShapedSurface}

GeologicStructure defining element defined by the morphology of a surface in the rock body; e.g. flute mark, slickenlines, fold.

\section{Derived from GSDefiningElement}

\section{SpatialObject}

A geometric entity and its location relative to some map horizon, projection, and scale. May be 2- or 3-D description of point, line, area, volume, etc. Depiction of the same feature on the Earth using different map projections, or at different scales is represented by multiple spatial objects in the database. $0 . .1$ cardinalities with Occurrence dictate that each of these depictions is a different Occurrence as well. 


\section{SpatialRelation}

A GeologicRelation between SpatialObjects Place holder class for specifying topologic relationships (containment, connectivity), and other kinds of relationships between SpatialObjects such as adjacency, proximity.

Derived from GeologicRelation

\section{StratigraphicAge}

A NamedAge defined as the time interval corresponding to a stratified chronostratigraphic unit. Corresponds to Geochronologic unit of NACSN [1983], article 80.

Derived from NamedAge

\section{StratigraphicColumn}

A graphic that depicts the stratigraphic relationships between GeologicUnits, with a vertical axis that represents time or thickness.

Derived from NamedAge

\section{StratigraphicRelation}

A GeologicRelation that involves the spatial, temporal, or hierarchical relationships between stratigraphic units.

\section{Derived from GeologicRelation}

\section{StructuralRelation}

A GeologicRelation that involves the spatial, temporal, or hierarchical relationships between two geologic structures or between a geologic structure and the rock units that encompass it.

\section{Derived from GeologicRelation}

\section{Symbol}

A collection of one or more graphical elements (color, pattern, design, character, etc.) used to represent an Occurrence or GeologicClass on a GeologicMap or CrossSection. Symbol can be thought of as an 'atomic' graphical unit, the simplest graphical unit that conveys meaning.

Derived from Graphic

\section{TemporalRelation}

A relationship involving GeologicStructure, GeologicUnit, Fossil, or GeologicProcess that explicitly state time relationships between objects. see Allen [1970] for discussion of possible temporal relationships.

\section{Derived from GeologicRelation}

\section{TextDescription}

TextDescription is an attribute of all GeologicConcepts. Therefore, any instance of any concept can be described by an arbitrary length text description. Intended as a location to store arbitrary length descriptions of individual instances of any concept. 


\section{TraceFossil}

Marks, trails, tracks, burrows, or other structural evidence of the behavior of an organism preserved in rocks or sediments. Generally does not include preserved or replaced remains, casts or molds.

\section{Derived from Fossil, GeologicStructure}

\section{UnconsolidatedMaterial}

A CompoundMaterial that consists of particles that are not bound together to form a solid material. May occur either at the surface or at depth. Includes sediment, soil, volcanic material, etc.

\section{Derived from CompoundMaterial}

\section{Universe}

A complete collection of all concepts. Forms the root class for all conceptual models, including NADM-C1. Logical TOP.

\section{Top concept in model}

\section{UnnamedAge}

GeologicAge interval specified by a younger and older bounding AgeDate, or by association with a particular geologic event. Equivalent to Geochronometric age of NACSN [1983]. Examples: 150-50 mya, contemporaneous with the xyz volcanic event.

\section{Derived from AgeInterval}

\section{VocabRelation}

A relationship between ControlledConcepts in a GeologicVocabulary. Used to specify hierarchy and other lexical relationships between terms in a GeologicVocabulary.

\section{Derived from GeologicRelation}

\section{VocabRole}

Identifies the role of a ControlledConcept in a VocabRelation. Specifies the standing of each ControlledConcept involved in a VocabRelation to the other ControlledConcepts participating in the relationship. Corresponds to 'AssociationEnd' names in a UML diagram. For example, in a hierarchy relationship between terms 'granitic rock' and 'granodiorite' in some GeologicVocabulary, VocabRelation is 'hierarchy', the VocabRole of 'granitic rock' in the relationship is 'parent', and the VocabRole of 'granodiorite' is 'child'.

\section{Derived from GeologicProperty}

\section{Void}

Empty space in a CompoundMaterial, e.g. pores, cavities, caves. Void space is considered a significant aspect of a lithologic description because of its importance in petroleum geology and hydrogeology. In early drafts of the model, void space was modeled through a VoidDescription object associated with CompoundMaterial. Representation of Void as a kind of EarthMaterial allows use of EMConstituent to describe void (pore) geometry (size and shape) and relationships (interconnectedness, relationship to solid constituents in rock) in the description of void space. 


\section{Volcanism}

The process by which magma and associated gases are extruded onto the Earth's surface and into the atmosphere. [revised from Jackson, 1997]

Derived from GeologicProcess

\section{WeatheringCharacter}

A description of the nature of the GeologicUnit at its interface with the atmosphere. Soil associated with a particular GeologicUnit would be described here, as would color and secondary mineral formation on a weathered surface.

\section{Derived from GeologicProperty}


Plate 1. Schema for top level geologic objects ......................................48

Plate 2. Schema for EarthMaterial..........................................................49

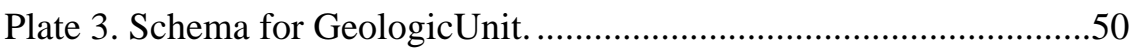

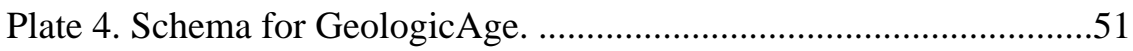

Plate 5. Schema for Fossil ..................................................................51

Plate 6 Schema for GeologicStructure ..................................................52

Plate 7. Schema for GeologicProcess and Genesis..................................53

Plate 8. Schema for GeologicRelation...................................................54

Plate 9. Schema for GeologicProperty ...................................................55

Plate 10. Schema for GeologicProperties related to ParticleGeometry ...56

Plate 11. Schema for GeologicVocabulary..........................................57

Plate 12. Schema for GeologicPortrayal .............................................57

Plate 13. Schema for GeologicPortrayalComponent..............................58

7.1 Notes on the models

The models presented here were constructed using Rational Rose software, and conform to UML 1.1. 


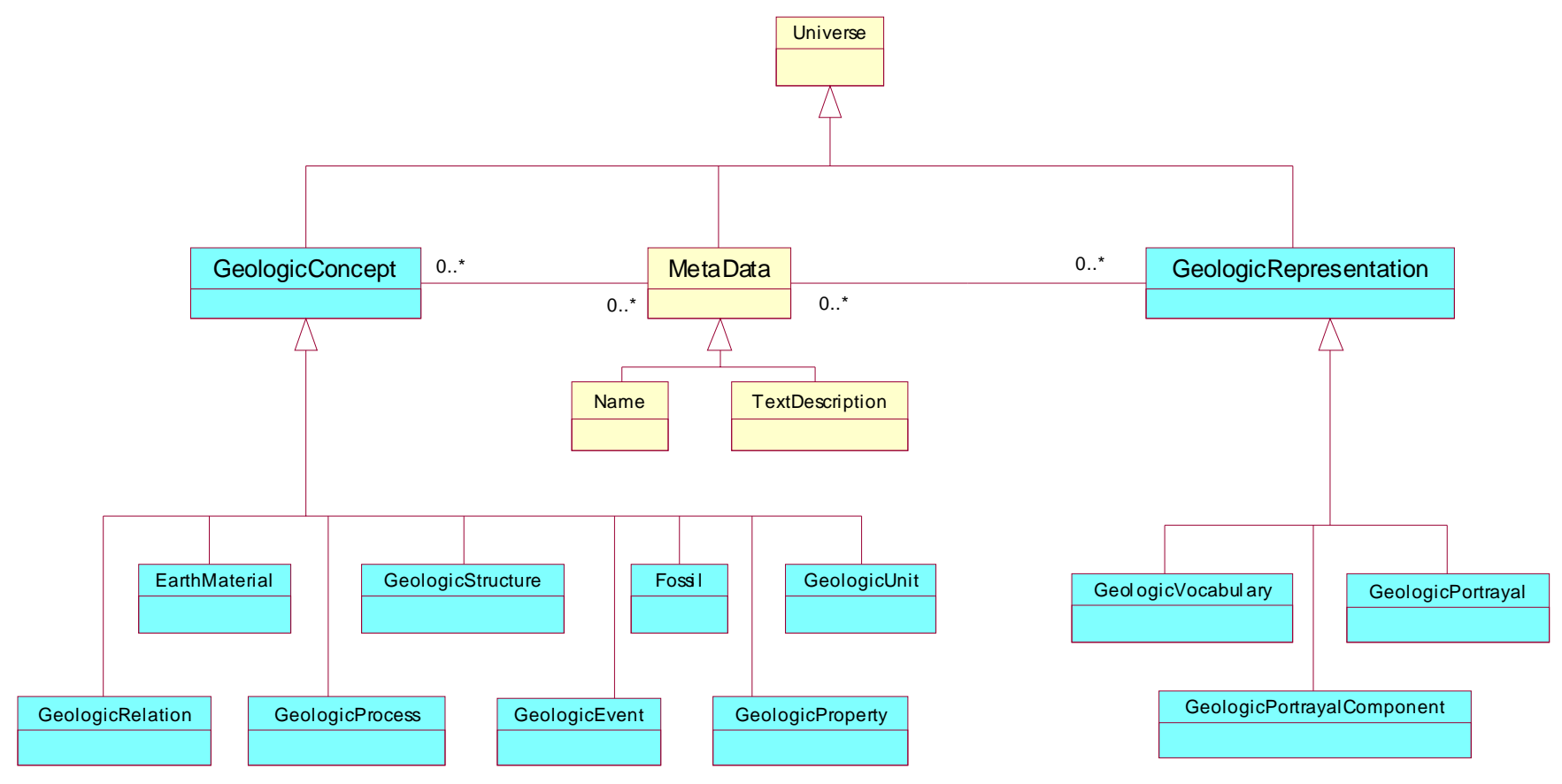

Plate 1. Schema for top level geologic objects 


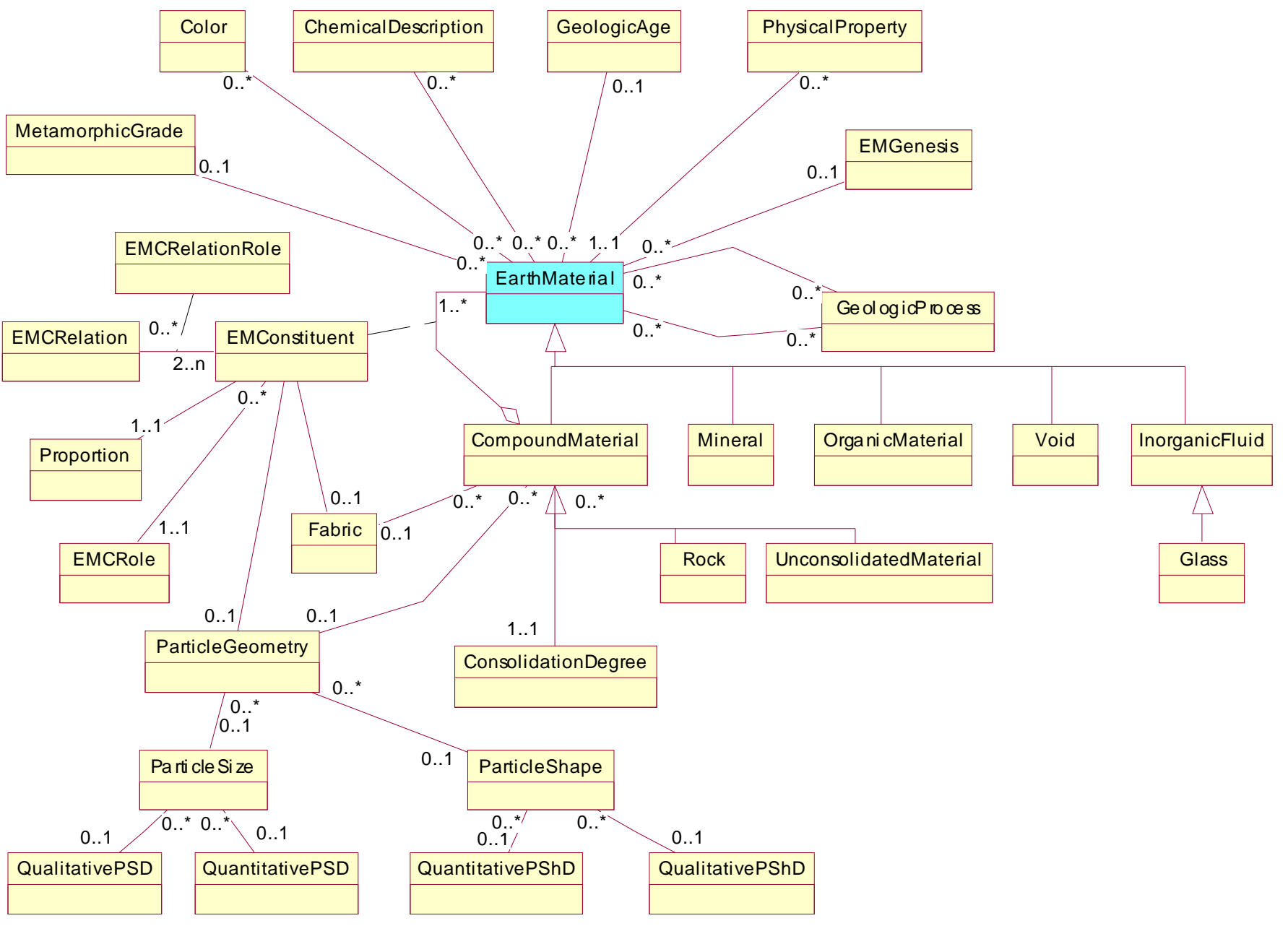

Plate 2. Schema for EarthMaterial 


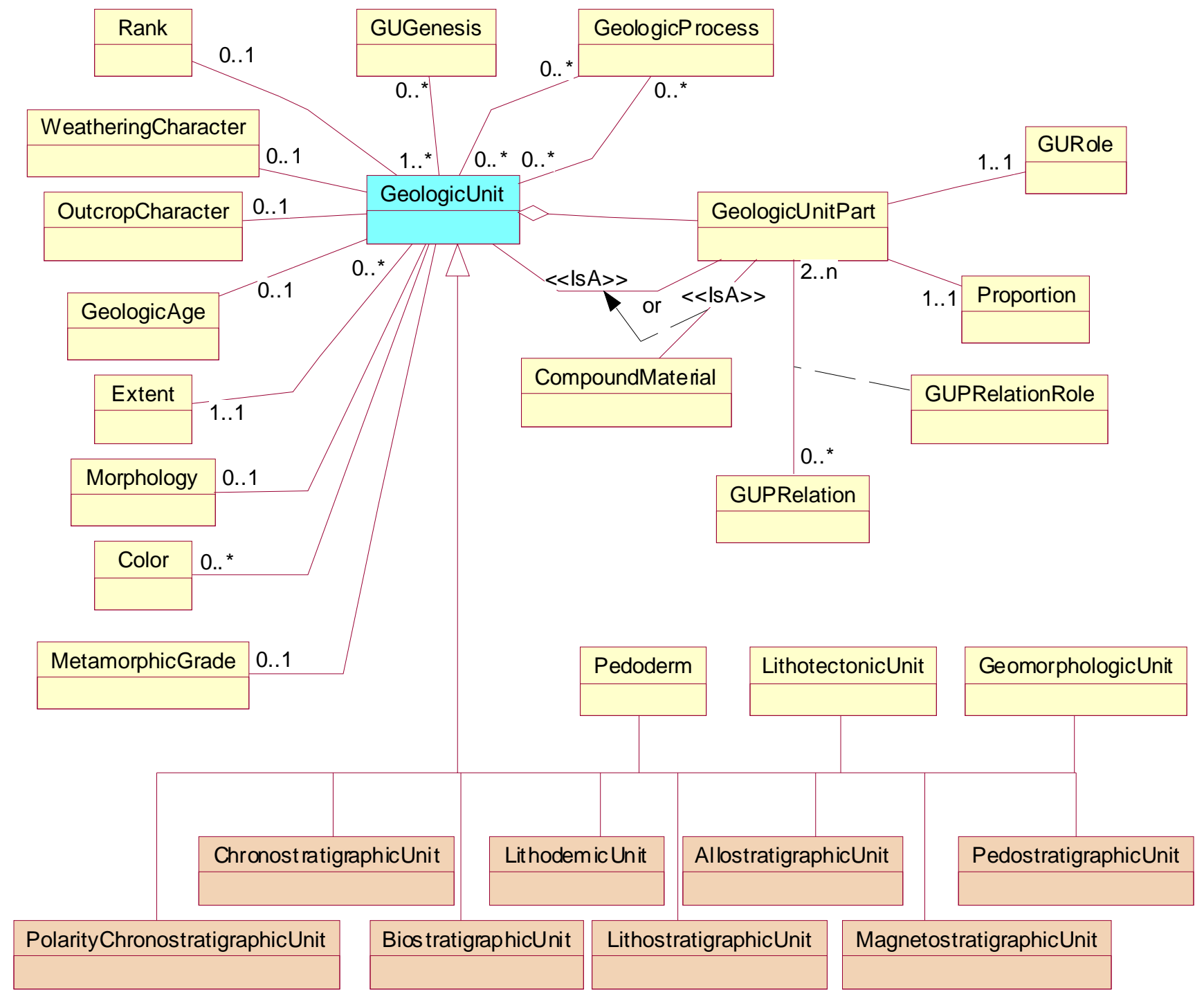

Plate 3. Schema for GeologicUnit 


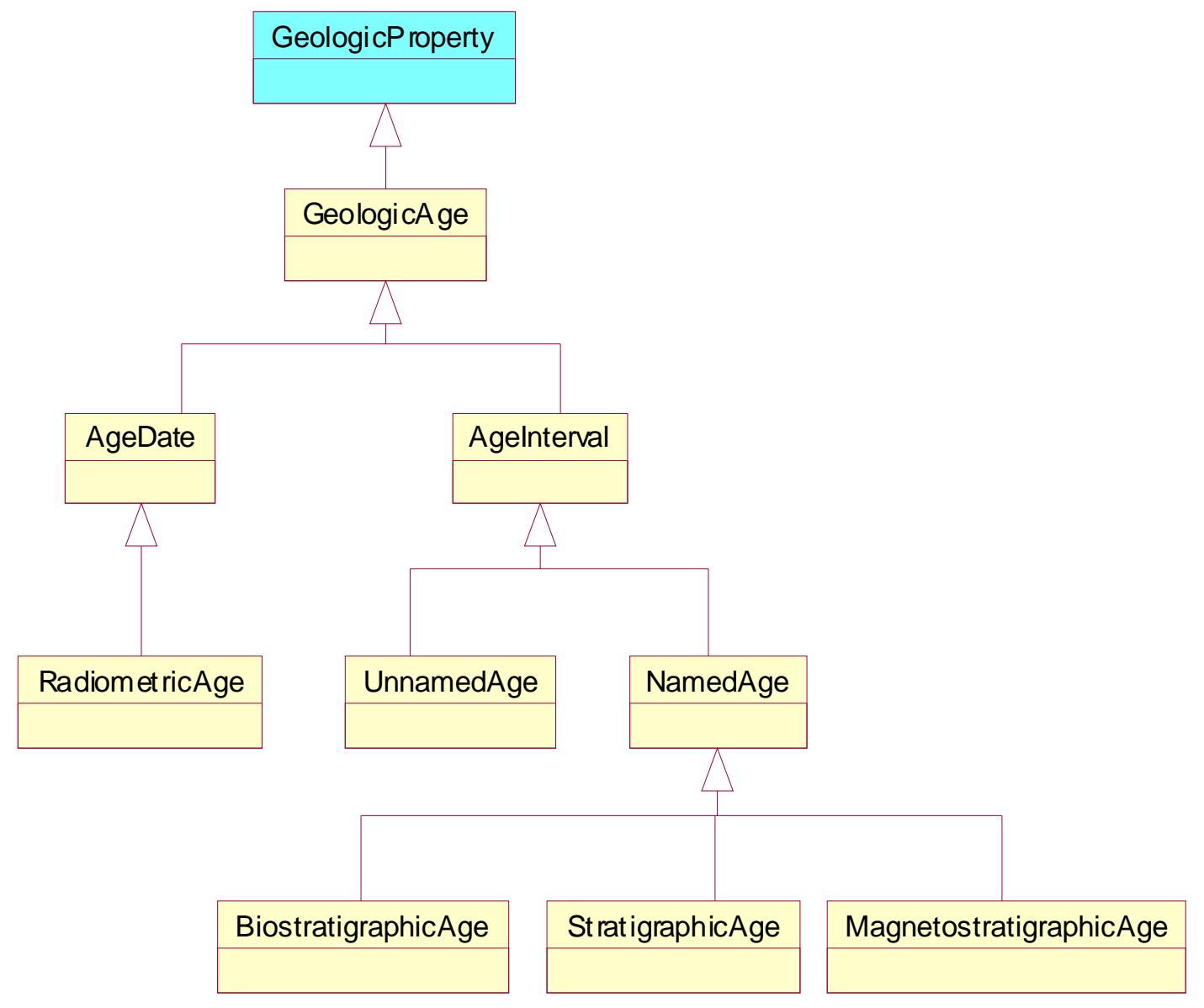

Plate 4. Schema for GeologicAge

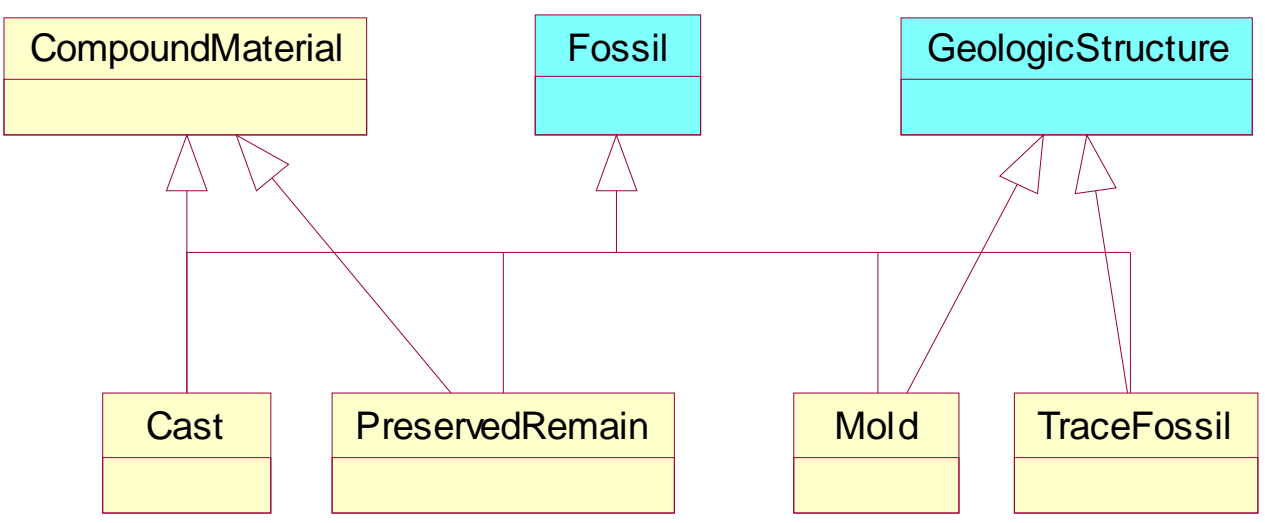

Plate 5. Schema for Fossil 


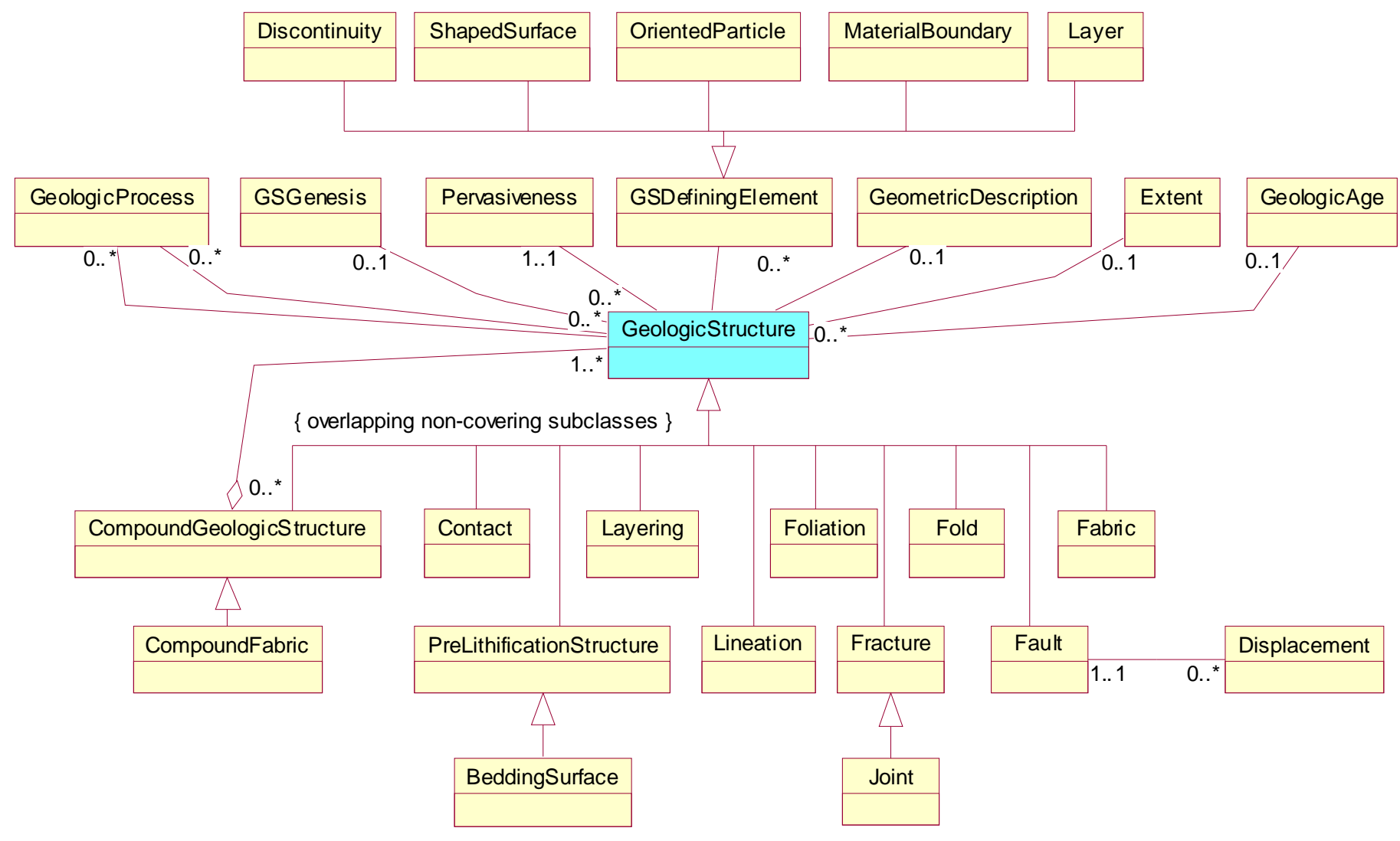

Plate 6. Schema for GeologicStructure 


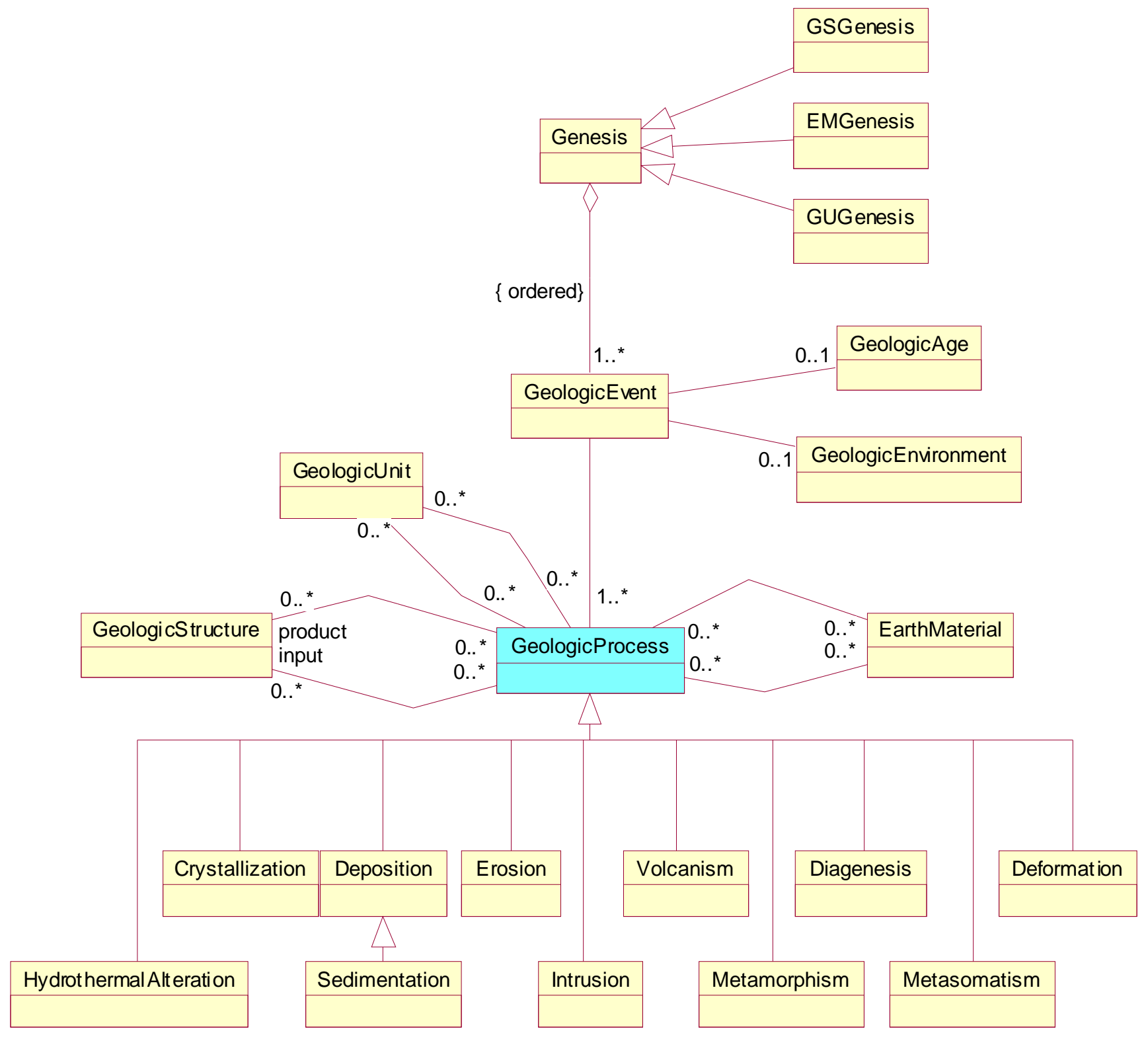

Plate 7. Schema for GeologicProcess and Genesis 


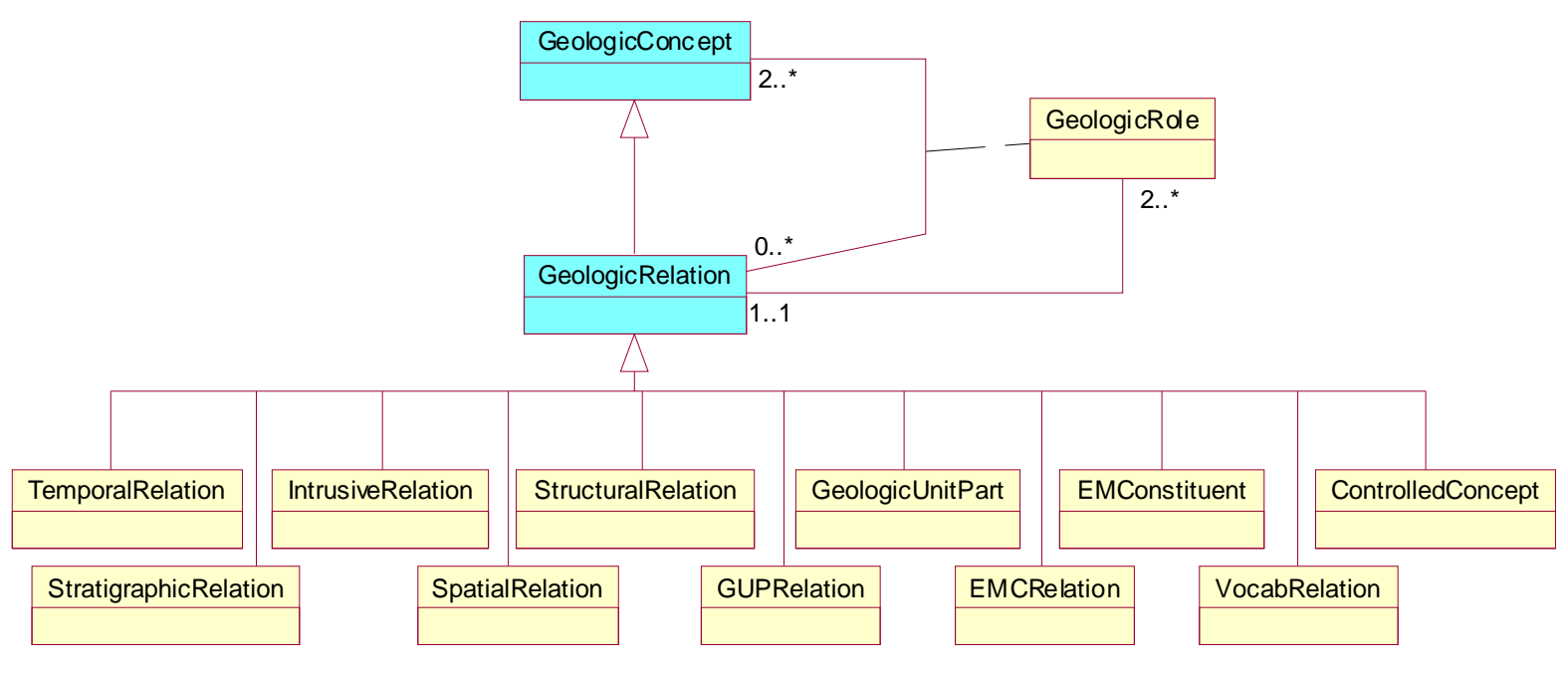

Plate 8. Schema for GeologicRelation 


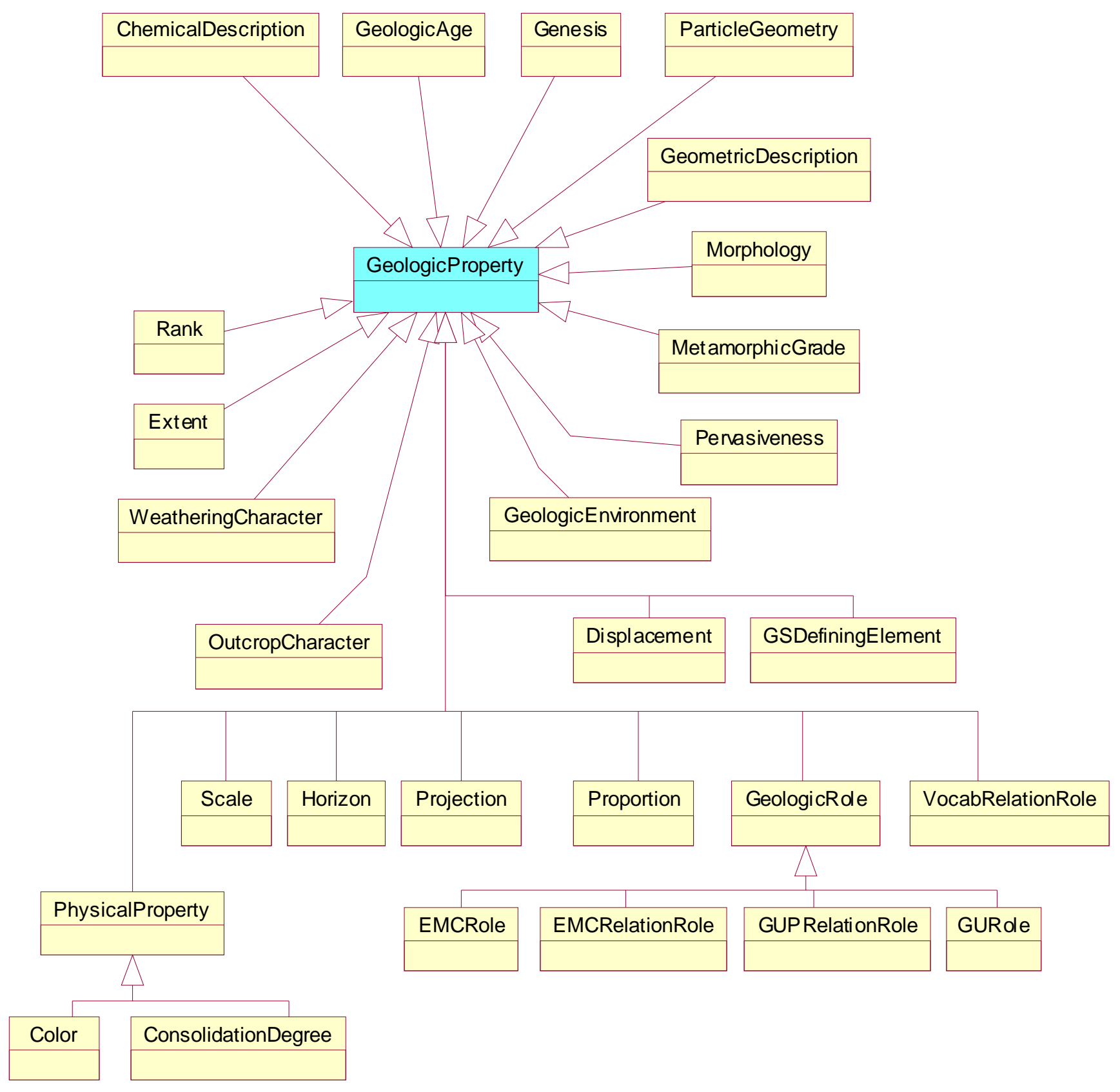

\section{Plate 9. Schema for GeologicProperty}




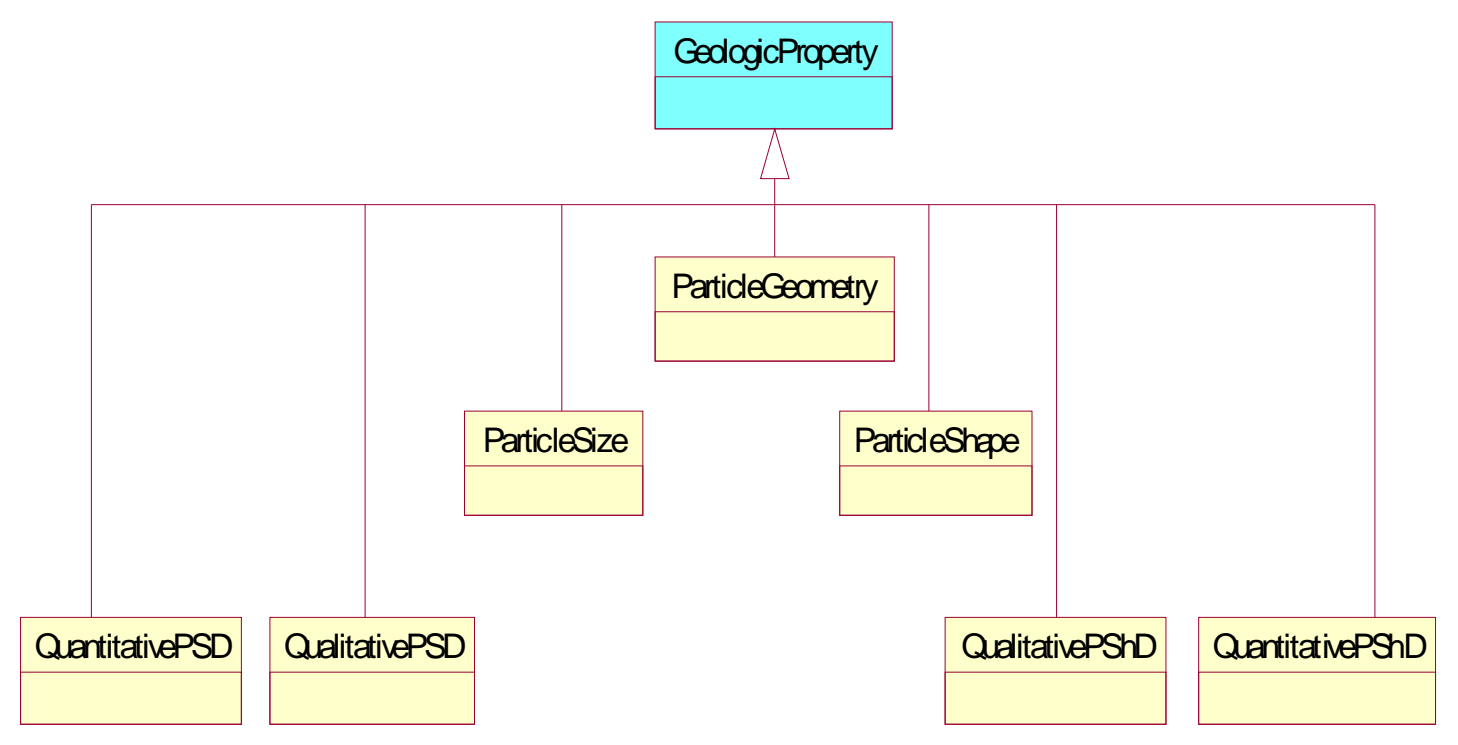

Plate 10. Schema for GeologicProperties related to ParticleGeometry

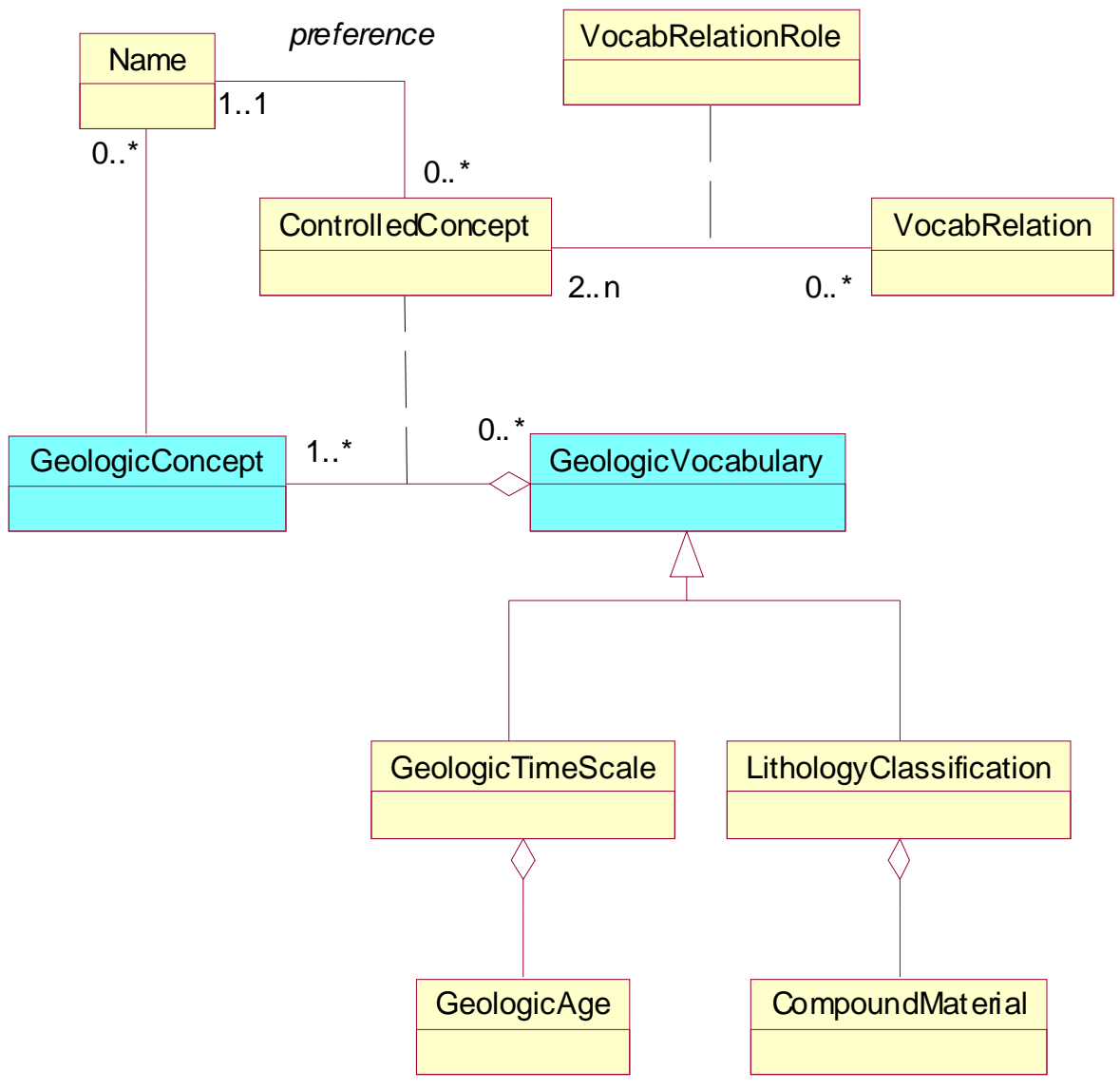

Plate 11. Schema for GeologicVocabulary 


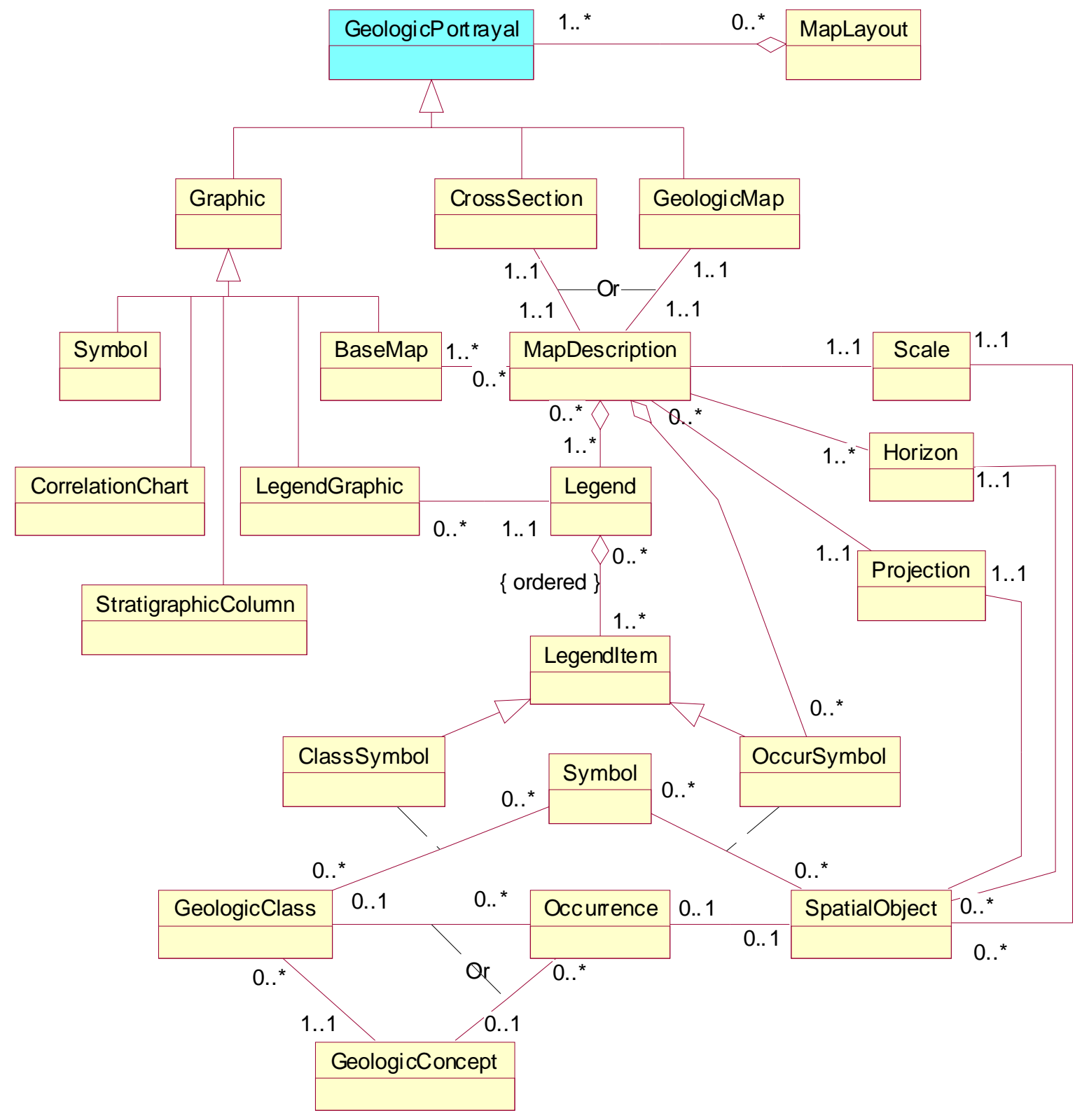

Plate 12. Schema for GeologicPortrayal 


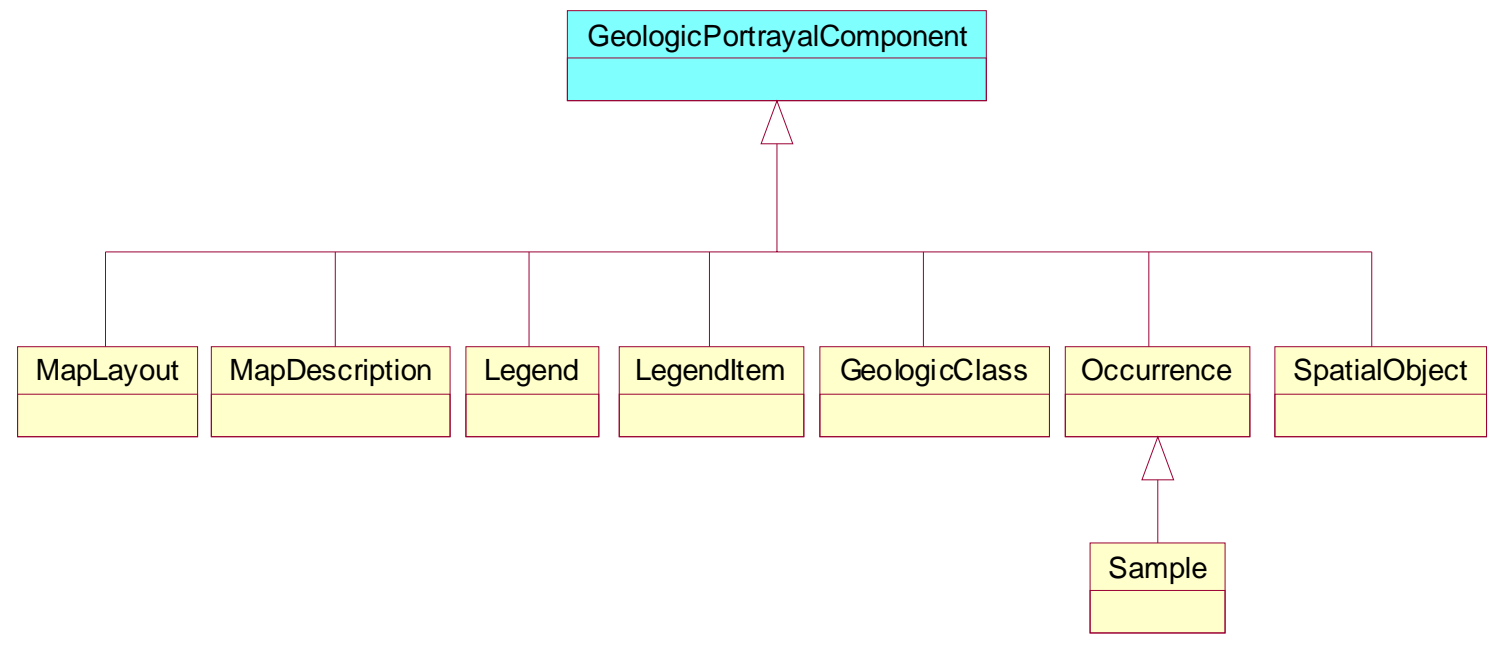

Plate 13. Schema for GeologicPortrayalComponent 
Available from:

Available from:

U.S. Geological Survey Information Services

Box 25286

Denver, $C O$

Also available at http://pubs.usgs.gov/of/2004/1334.

Geological Survey of Canada

601 Booth Street

Ottawa, Ontario K1A 0E8

Also available at http://www.nrcan.gc.ca/gsc/bookstore.

Display copy available at Geological Survey of Canada libraries.

Also available at http://nadm-geo.org/.

System requirements:

The document version of this Open File report can be viewed using Adobe Acrobat Reader (not included). 